\title{
Slow-, Tight-Binding Inhibition of CYP17A1 by Abiraterone Redefines Its Kinetic Selectivity and Dosing Regimen ${ }^{\mathbb{S}}$
}

\author{
Eleanor Jing Yi Cheong, Pramod C. Nair, Rebecca Wan Yi Neo, Ho Thanh Tu, Fu Lin, \\ Edmund Chiong, Kesavan Esuvaranathan, Hao Fan, Russell Z. Szmulewitz, Cody J. Peer, \\ () William D. Figg, Christina Li Lin Chai, John O. Miners, and (1)Eric Chun Yong Chan
}

Department of Pharmacy, Faculty of Science (E.J.Y.C., R.W.Y.N., H.T.T., C.L.L.C., E.C.Y.C.) and Department of Biological Sciences (H.F.), National University of Singapore, Singapore, Singapore; Department of Clinical Pharmacology and Flinders Centre for Innovation in Cancer, College of Medicine and Public Health, Flinders University, Adelaide, Australia (P.C.N., J.O.M.); Bioinformatics Institute, Biotransformation Innovation Platform (BioTrans) (F.L.) and Bioinformatics Institute (H.F.), Agency for Science, Technology and Research (A*STAR), Singapore, Singapore; Department of Surgery, National University Health System, Singapore, Singapore (E.C., K.E.); Department of Urology, National University Hospital, Singapore, Singapore (E.C., K.E.); Centre for Computational Biology, DUKE-NUS Medical School, Singapore, Singapore (H.F.); The University of Chicago, Chicago, Illinois (R.Z.S.); National Cancer Institute, Rockville, Maryland (C.J.P., W.D.F.); and National University Cancer Institute, Singapore (NCIS), NUH Medical Centre (NUHMC), Singapore, Singapore (E.C.Y.C.)

Received February 20, 2020; accepted June 11, 2020

\section{ABSTRACT}

Substantial evidence underscores the clinical efficacy of inhibiting CYP17A1-mediated androgen biosynthesis by abiraterone for treatment of prostate oncology. Previous structural analysis and in vitro assays revealed inconsistencies surrounding the nature and potency of CYP17A1 inhibition by abiraterone. Here, we establish that abiraterone is a slow-, tight-binding inhibitor of CYP17A1, with initial weak binding preceding the subsequent slow isomerization to a high-affinity CYP17A1-abiraterone complex. The in vitro inhibition constant of the final high-affinity CYP17A1-abiraterone complex $\left(\left(K_{i}^{*}=0.39 n M\right)\right.$ yielded a binding free energy of $-12.8 \mathrm{kcal} / \mathrm{mol}$ that was quantitatively consistent with the in silico prediction of $-14.5 \mathrm{kcal} / \mathrm{mol}$. Prolonged suppression of dehydroepiandrosterone (DHEA) concentrations observed in VCaP cells after abiraterone washout corroborated its protracted CYP17A1 engagement. Molecular dynamics simulations illuminated potential structural determinants underlying the rapid reversible binding characterizing the two-step induced-fit model. Given the extended residence time (42 hours) of abiraterone within the CYP17A1 active site, in silico simulations demonstrated sustained target engagement even when most abiraterone has been eliminated systemically. Subsequent pharmacokineticpharmacodynamic (PK-PD) modeling linking time-dependent CYP17A1 occupancy to in vitro steroidogenic dynamics predicted comparable suppression of downstream DHEA-sulfate at both 1000- and 500-mg doses of abiraterone acetate. This enabled mechanistic rationalization of a clinically reported PK-PD disconnect, in which equipotent reduction of downstream plasma DHEAsulfate levels was achieved despite a lower systemic exposure of abiraterone. Our novel findings provide the impetus for reevaluating the current dosing paradigm of abiraterone with the aim of preserving PD efficacy while mitigating its dose-dependent adverse effects and financial burden.

\section{SIGNIFICANCE STATEMENT}

With the advent of novel molecularly targeted anticancer modalities, it is becoming increasingly evident that optimal dose selection must necessarily be predicated on mechanistic characterization of the relationships between target exposure, drug-target interactions, and pharmacodynamic endpoints. Nevertheless, efficacy has always been perceived as being exclusively synonymous with affinity-based measurements of drug-target binding. This work demonstrates how elucidating the slow-, tight-binding inhibition of CYP17A1 by abiraterone via in vitro and in silico analyses was pivotal in establishing the role of kinetic selectivity in mediating time-dependent CYP17A1 engagement and eventually downstream efficacy outcomes.

\section{Introduction}

Prostate cancer is ranked as the second most frequent cancer and the fifth leading cause of death among male

This work was supported by the Singapore Ministry of Education Tier 1 Academic Research Funding [Grant R-148-000-249-114] and the National University of Singapore President's Graduate Fellowship to E.J.Y.C. and the National University of Singapore, Department of Pharmacy, Final Year Project Funding provided to R.W.Y.N. F.L. and H.F. gratefully acknowledge the financial support from the Biomedical Research Council of the Agency for Science, Technology and Research, Singapore.

https://doi.org/10.1124/jpet.120.265868.

S This article has supplemental material available at jpet.aspetjournals.org. malignancies worldwide (Bray et al., 2018). Therapeutic interventions in prostate cancer are defined upon patient stratification into the clinical states continuum (Scher et al., 2016). Although localized prostate cancer remains amenable to curative interventions via radiation or surgery, androgen deprivation therapy (ADT) in the form of surgical or medical castration has become the standard of care in clinically advanced or disseminated disease. However, the initial response to ADT is often unsustainable, and patients inevitably experience progression to castration-resistant prostate cancer within 2 to 3 years (Pienta and Bradley, 2006; Harris et al., 2009). Metastases (mCRPC) are evident in $\geq 84 \%$ of diagnosed 
cases, and prognosis is poor in MCRPC. Substantial evidence alludes to the reactivation of androgen receptor (AR)-mediated signaling as a key driver in disease progression despite castrate serum testosterone $(<50 \mathrm{ng} / \mathrm{dl})$ levels (Mostaghel et al., 2007). As a result, secondary hormonal manipulation via targeted molecular therapies has gained traction in the therapeutic management of mCRPC.

Bifunctional CYP17A1 occupies a pivotal role in both adrenal and de novo intratumoral androgen biosynthesis in which it catalyzes sequential $17 \alpha$-hydroxylation and C17,20-lyase reactions (Fig. 1A) (Porubek, 2013). The resulting product, dehydroepiandrosterone (DHEA), is a critical precursor for the downstream generation of potent $\mathrm{AR}$ ligands testosterone and dihydrotestosterone. Consequently, abiraterone [administered as prodrug abiraterone acetate (AA)] was first developed and approved in 2011 as a first-in-class CYP17A1 inhibitor to treat mCRPC (Vasaitis et al., 2011; Yin and Hu, 2014). More recently, in men with metastatic hormone-sensitive prostate cancer (mHSPC), AA in combination with ADT was shown to significantly increase overall survival as compared with ADT monotherapy, culminating in the expansion of the clinical indications of AA to include the population of patients with mHSPC (Fizazi et al., 2017).

AA is currently indicated to be administered at a dose of $1000 \mathrm{mg}$ daily either 1 hour before or 2 hours after food (Janssen, 2019). Given that no dose-limiting toxicities were detected up to $2000 \mathrm{mg}$ in a phase I dose escalation trial, the current dose selection was justified based on a plateau in anticipated toxicities (i.e., upstream mineralocorticoid excess) observed at doses above $750 \mathrm{mg}$ (Attard et al., 2008). Implicit in this classic maximal tolerated dose (MTD) paradigm are the principles that 1) a linear dose-efficacy relationship exists and 2) toxicities are direct manifestations of exacerbated pharmacology (Ji et al., 2018). However, in a recent phase II trial, comparisons of low-dose AA (250 mg with a low-fat meal) versus standard-dose AA (1000 mg fasting) demonstrated that despite trough abiraterone concentrations being significantly higher in the standard-dose group compared with the low-dose group, the extent of dehydroepiandrosterone-sulfate (DHEAS) suppression was similar, verifying that the efficacy of CYP17A1 inhibition was preserved (Szmulewitz et al., 2018). This observed pharmacokinetic-pharmacodynamic (PK-PD) uncoupling underscores how targeted therapeutics such as AA may achieve optimal antitumor activity at doses significantly lower than required to elicit adverse outcomes. With the diminished utility of MTD-based strategies, rational dose selection for AA must instead be predicated on mechanistic and quantitative characterization of the relationships between target exposure, drug-target interactions, and PD endpoints (Minasian et al., 2014; Sachs et al., 2016).

However, the mechanism of CYP17A1 inhibition by abiraterone has not been fully characterized. Firstly, X-ray crystal structures and spectral ligand binding assays reported coordination of the C17 pyridine of abiraterone with CYP17A1 heme iron, indicating a potent but reversible type II interaction (DeVore and Scott, 2012). Garrido et al. (2014) postulated that abiraterone is a slowly reversible CYP17A1 inhibitor and demonstrated its tight-binding effect with jump dilution experiments. Furthermore, abiraterone was suggested to be a slow-binding inhibitor with enhanced inhibition upon preincubation with CYP17A1 (Jarman et al., 1998). Collectively, the apparent complexities in the inhibition kinetics of CYP17A1 by abiraterone allude to a potential slow-, tight-binding phenomenon (Fig. 1B). Consequently, both inhibitory potency (thermodynamic selectivity) and target occupancy (kinetic selectivity) by abiraterone against CYP17A1 must be explicitly considered when defining its in vivo drug activity.

Moreover, it was revealed that $\Delta^{4}$-abiraterone (D4A) (Fig. 1C), a downstream metabolite of abiraterone, inhibits multiple steroidogenic enzymes, including CYP17A1 (Li et al., 2015). The multitargeting effects of D4A, coupled with its direct $A R$ antagonism, have underscored its potential clinical utility in prostate oncology. Considering the structural similarities between abiraterone and D4A, particularly the preservation of the pyridine ring, the inhibition kinetics of CYP17A1 by D4A are expected to be equally complex.

In this study, detailed in vitro biochemical and in silico molecular dynamics (MD) analyses allowed us to gain unprecedented insights into the kinetic and structural determinants of the abiraterone/D4A-CYP17A1 interaction. Utilizing a mechanistic PK-PD model, we subsequently demonstrated how the coupling between $\mathrm{PK}$ and the extended residence time of abiraterone within the CYP17A1 active site potentially plays a pivotal role in controlling time-dependent CYP17A1 occupancy and eventually downstream PD outcomes. Our findings could eventually guide the optimization of AA dosing across the continuum of prostate oncology.

\section{Methods}

Chemicals and Reagents. Abiraterone and DHEA were purchased from Tokyo Chemical Industry (Tokyo, Japan), whereas D4A was synthesized in-house (Supplemental Fig. 1; Supplemental Methods). Prednisolone, progesterone, 17 $\alpha$-hydroxyprogesterone, $17 \alpha$-hydroxypregnenolone, androstenedione, testosterone- $2,3,4-{ }^{13} \mathrm{C}_{3}$, ketoconazole, and hydroxylamine hydrochloride were purchased from Sigma-Aldrich (St. Louis, MO). NADPH-regenerating system consisting of NADPH A (NADP+ and glucose 6-phosphate) and NADPH B (glucose-6 phosphate dehydrogenase) was purchased from BD Gentest (Woburn, MA). Dulbecco's Modified Eagle's medium high glucose, fetal bovine serum and $10 \times$ trypsin-EDTA were purchased from Gibco Life Technologies (Waltham, MA). One molar stock solution of phosphate buffer saline was purchased from Vivantis (Subang Jaya, Malaysia). All other analytical reagents were of analytical grade.

Enzymes. Human CYP17A1R bactosomes containing recombinant human CYP17A1 (rCYP17A1) and human cytochrome P450-

\footnotetext{
ABBREVIATIONS: AA, abiraterone acetate; ADT, androgen deprivation therapy; AR, androgen receptor; ATCC, American Type Culture Collection; AUC area under the plasma concentration-time curve.Cl, confidence interval; COM, center of mass; D4A, $\Delta^{4}$ abiraterone; DHEA, dehydroepiandrosterone; DHEA-S, dehydroepiandrosterone-sulfate; El, initial enzyme-inhibitor encounter complex; El ${ }^{*}$, the final high-affinity enzyme-inhibitor complex; $k_{1}$, rate constant for the initial binding of inhibitor to enzyme; $k_{2}$, rate constant for the dissociation of the initial $E I$ complex; $k_{3}$, forward isomerization rate constant for the conversion of $E l$ to $E l^{\star} ; k_{4}$, reverse isomerization rate constant for the conversion of $E l^{\star}$ to $E l$; $K_{i}$, inhibition constant for the initial $E l$ complex; $K_{i}^{*}$, inhibition constant for the final $E l^{*}$ complex; $K_{i, \text { app }}$, apparent inhibition constant for the initial $E I$ complex; $K_{\text {obs }}$, first-order rate constant for onset of inhibition; LC/MS/MS, liquid chromatography tandem mass spectrometry; mCRPC, metastatic castration-resistant prostate cancer; MD, molecular dynamics; mHSPC, metastatic hormone-sensitive prostate cancer; MTD, maximal tolerated dose; PD, pharmacodynamics; PK, pharmacokinetics; PMF, potential of mean force; rCYP17A1, recombinant CYP17A1; $t_{1 / 2}$, half-life.
} 


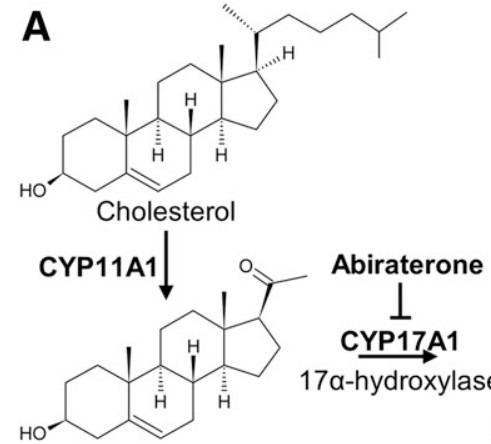

Pregnenolone

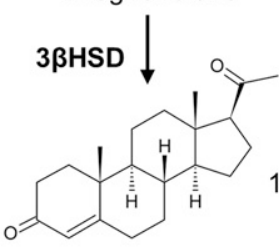

Progesterone

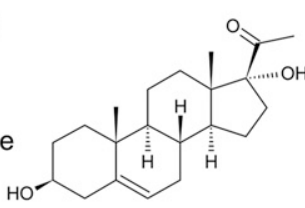

17a-hydroxypregnenolone

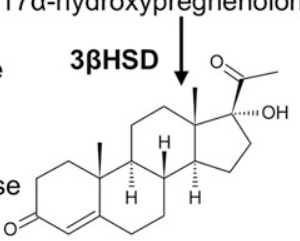

17a-hydroxyprogesterone

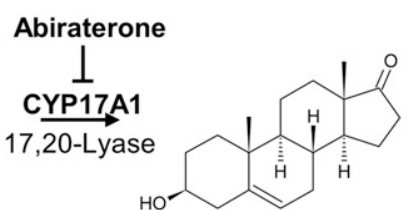

Dehydroepiandrosterone

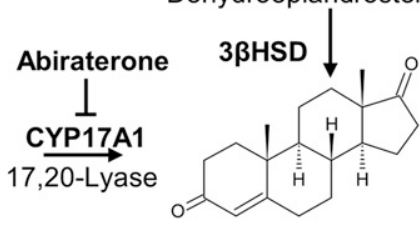

Androstenedione

B
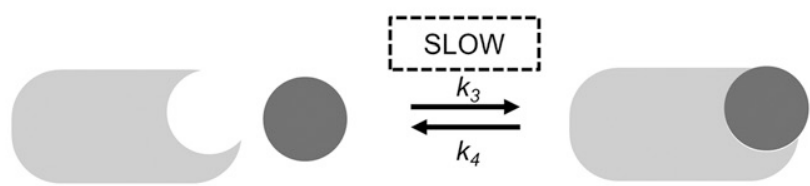

CYP17A1 (E) Abiraterone/D4A (I)

El

CYP17A1 (E) Abiraterone/D4A (I)

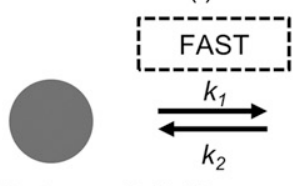

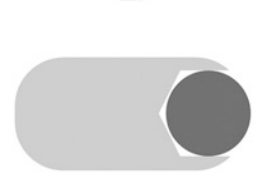

EI

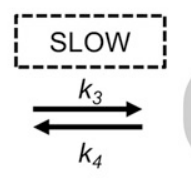

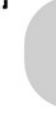

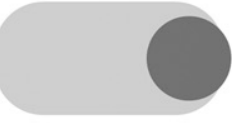

$\mathrm{El}^{*}$
Fig. 1. Clinical utility of abiraterone and D4A in prostate cancer. (A) Endogenous androgen biosynthesis pathway showing the inhibitory effects of abiraterone/D4A on CYP17A1. (B) Schematic describing the two proposed mechanisms of slow-, tight-binding inhibition of CYP17A1 by abiraterone/D4A (C) Chemical structures of abiraterone and $\mathrm{D} 4 \mathrm{~A}$ in which the latter is formed via metabolism of abiraterone by $3 \beta$-hydroxysteroid dehydrogenase $(3 \beta \mathrm{HSD})$.

\section{C}

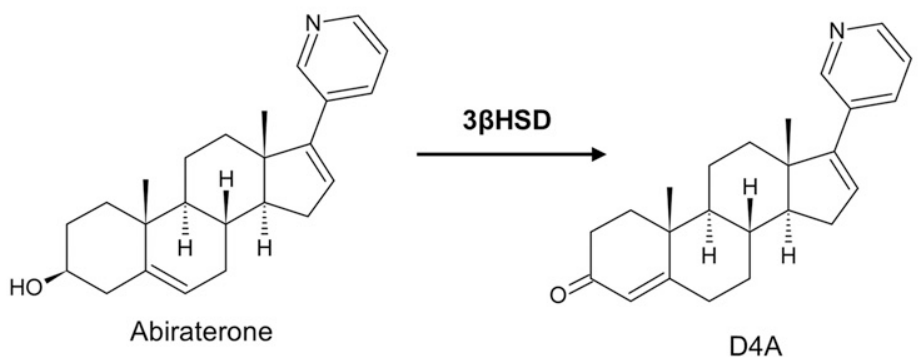

reductase enzymes supplemented with purified human cytochrome b5 were purchased from Cypex (Dundee, UK).

Slow-Binding Inhibition Kinetics. To characterize the slowbinding inhibition of CYP17A1 by abiraterone and D4A, preincubation time dependence of reaction velocity was measured (Morrison and Walsh, 1988). Given the dual catalytic pathways associated with CYP17A1, both inhibition of progesterone $17 \alpha$-hydroxylation and $17 \alpha$ hydroxypregnenolone C17,20-lyase reactions were evaluated. Incubations $(n=3)$ were performed in 96-well plates. Abiraterone or D4A (5-100 nM) was preincubated for $0-30$ minutes at $37^{\circ} \mathrm{C}$ with rCYP17A1 $(5 \mathrm{pmol} / \mathrm{ml})$ and NADPH B in $50 \mathrm{mM}$ potassium phosphate buffer ( $\mathrm{pH}$ 7.4) containing $2 \mathrm{mM} \mathrm{MgCl} 2$. Reactions were initiated by the addition of NADPH A and either $50 \mu \mathrm{M}$ progesterone or $5 \mu \mathrm{M} 17 \alpha$ hydroxypregnenolone (saturating substrate conditions; Supplemental Fig. 2) and were allowed to proceed over the previously optimized incubation time of 20 minutes (Supplemental Fig. 2; Supplemental Methods). Eighty-microliter aliquots were subsequently removed and quenched with an equal volume of ice-cold acetonitrile containing the internal standard (prednisolone and androstenedione were used in the $17 \alpha$-hydroxylase and C17,20-lyase inhibition assays, respectively). The quenched samples were centrifuged at $2755 \mathrm{~g}, 4^{\circ} \mathrm{C}$, for 30 minutes, and the supernatant fraction was subjected to liquid chromatography tandem mass spectrometry (LC/MS/MS) analysis to determine the formation of $17 \alpha$-hydroxyprogesterone or DHEA oxime, which were monitored as measures of residual enzyme activity. Oxime derivatization of DHEA and androstenedione prior to LC/MS/MS analysis is further described in the Supplemental Methods and Supplemental Fig. 3.

Semilogarithmic plots illustrating the percentage of rCYP17A1 activity remaining $\left(v / v_{0}\right)$ (relative to an $[I]=0$ preincubation control sample) 
against preincubation time $(t)$ were generated for the determination of the observed first-order rate constant for the onset of inhibition $\left(k_{o b s}\right)$ at various inhibitor concentrations (eq. 1) (Copeland, 2000, 2013).

$$
\ln \left(\frac{v}{v_{0}}\right)=-k_{o b s} \cdot \mathrm{t}
$$

Estimation of $K_{i}, a p p$ for the Initial Encounter Complex. To obtain the apparent inhibition constant $\left(K_{i}, a p p\right)$ for the initial CYP17A1-inhibitor encounter complex, procedures were similar to that of the preincubation time-dependent inhibition assays, albeit without preincubation. The measured initial velocity $\left(v_{i}\right)$ across various concentrations of abiraterone and D4A (1-300 $\mathrm{nM})$ was normalized against the velocity of the uninhibited reaction $\left(v_{0}\right)$. Data were fitted using standard four-parameter logistic models in GraphPad Prism 7.04 (San Diego, CA) as delineated in eq. 2 to generate $K_{i}$, app (Copeland, 2013).

$$
\frac{v_{i}}{v_{0}}=\frac{1}{1+\frac{[I]}{K_{i}, a p p}}
$$

Determination of $\boldsymbol{K}_{i}^{*}$ for the $\boldsymbol{E I}^{*}$ Complex. Using nonlinear regression in GraphPad Prism 7.04, the measured $k_{o b s}$ against [I] was analyzed via a two-step inhibition mechanism as shown in Fig. 1B, in which the initial rapid binding of inhibitor to enzyme $[E I]$ is accompanied by subsequent slower isomerization to the final enzyme-inhibitor $\left[E I^{*}\right]$ complex (Copeland, 2013). Equation 3a was used to obtain the forward $\left(k_{3}\right)$ and reverse kinetic constants $\left(k_{4}\right)$, describing the slow-binding inhibition. Given that $k_{4}$ reflects the rate-limiting step for enzyme reactivation in the case of slow binding, the dissociation half-life for slow-binding inhibitors could be calculated using eq. 3b (Copeland, 2013).

$$
\begin{gathered}
k_{o b s}=k_{4}+\frac{k_{3}[I]}{K_{i}, a p p+[I]} \\
\text { Dissociation } t_{1 / 2}=\frac{\ln 2}{k_{o b s}} \approx \frac{\ln 2}{k_{4}}
\end{gathered}
$$

To determine the true values of $K_{i}$ from experimental $K_{i}, a p p$, reversible inhibition assays were performed, and Lineweaver-Burk transformations were used to discern the mode of inhibition (Supplemental Methods). Lastly, the inhibition constants $\left(K_{i}^{*}\right)$ of the $E I^{*}$ complex, representing the true target affinity of inhibitor to CYP17A1, were determined using eq. 4 (Copeland, 2013).

$$
K_{i}^{*}=\frac{K_{i} k_{4}}{k_{3}+k_{4}}
$$

Verifying the Functionality of the C17,20 Lyase Pathway in VCaP Cells. VCaP prostate cancer cells (ATCC CRL-2876) were purchased from American Type Culture Collection (ATCC) (Manassas, VA) and authenticated by ATCC. VCaP cells were cultured in Dulbecco's modified Eagle's medium media supplemented with $10 \% \mathrm{v} / \mathrm{v}$ fetal bovine serum. For kinetic analyses, cells (passage numbers 8-16) were seeded in 24 -well plates at a density of 0.4 million cells per well in a volume of $500 \mu$ l. Upon confluency, culture medium was first removed, and each well was washed with phosphate buffer saline to minimize residual metabolic waste. Cells were incubated with media containing $17 \alpha$ hydroxypregnenolone (2.5-45 $\mu \mathrm{M}, 1 \% \mathrm{v} / \mathrm{v}$ methanol). After 30 minutes, $200-\mu \mathrm{l}$ aliquots of medium were collected, spiked with internal standard, and subjected to a two-step liquid-liquid extraction using methyl-tert butyl ether before concentration under nitrogen gas (Supplemental Methods). The dried residues were derivatized via reconstitution with $50 \mu \mathrm{l}$ of $100 \mathrm{mM}$ hydroxylamine hydrochloride solution $(50 \% \mathrm{v} / \mathrm{v}$ methanol/water) and heated at $60^{\circ} \mathrm{C}$ for 1 hour prior to LC/MS/MS measurement of DHEA oxime formation (Ming et al., 2014). Subsequent assessment of inhibitor functionality involved the simultaneous coincubation of inhibitors (abiraterone: $20 \mu \mathrm{M}, 0.5 \% \mathrm{v} / \mathrm{v}$ methanol; ketoconazole: $25 \mu \mathrm{M}, 0.5 \% \mathrm{v} / \mathrm{v} \mathrm{DMSO})$ or vehicle control (0.5\% v/v methanol/DMSO), along with probe substrate ( $17 \alpha$-hydroxypregnenolone: $30 \mu \mathrm{M}, 0.6 \% \mathrm{v} / \mathrm{v}$ methanol) for 2 hours before analysis of DHEA oxime formation.

Functional Recovery Experiments in VCaP Cells. Preincubation experiments utilizing $\mathrm{VCaP}$ cells were initiated by the addition of media containing abiraterone $(40 \mu \mathrm{M}, 1 \% \mathrm{v} / \mathrm{v}$ methanol), known reversible CYP17A1 inhibitor ketoconazole ( $40 \mu \mathrm{M}, 1 \% \mathrm{v} / \mathrm{v}$ DMSO), along with vehicle controls (1\% v/v methanol/DMSO). After 2 hours, inhibitors were removed, and fresh medium was added for the duration of the washout period ( 0,30 , or 60 minutes). Finally, medium containing the probe substrate $17 \alpha$-hydroxypregnenolone ( $30 \mu \mathrm{M}, 1 \% \mathrm{v} / \mathrm{v}$ methanol) was added for 30 minutes. Two-hundred-microliter aliquots of medium were collected and processed in the same manner as described in the previous section to measure DHEA oxime formation. At each time point ( 0,30 , or 60 minutes), unpaired one-tailed $t$ tests were applied to evaluate whether the percentage recovery of maximal CYP17A1 activity (quantified by DHEA oxime formation in the respective vehicle controls) after abiraterone preincubation and washout was significantly suppressed in comparison with ketoconazole treatment $(P$ value $<0.05)$.

LC/MS/MS Measurement of Metabolite Formation. Samples were analyzed using an Agilent 1290 Infinity liquid chromatography system (Agilent Technologies, Santa Clara, CA) interfaced with an AB Sciex QTrap 5500 hybrid linear ion-trap quadrupole mass spectrometer equipped with a TurboIonSpray source (AB SCIEX, Framingham, MA). Injection volumes were 2 and $4 \mu$ l when quantifying $17 \alpha$ hydroxyprogesterone and DHEA oxime production, respectively. AWaters ACQUITY ${ }^{\mathrm{TM}}$ ultra-high performance liquid chromatography ethylene bridged hybrid $\mathrm{C}_{18}$ column $(1.7 \mu \mathrm{M}, 2.1 \times 50 \mathrm{~mm}$; Waters, Milford) was used for chromatographic separation. The aqueous mobile phases comprised $0.1 \% \mathrm{v} / \mathrm{v}$ formic acid in water (A) and $0.1 \% \mathrm{v} / \mathrm{v}$ formic acid in acetonitrile (B). Mobile phases were delivered at $0.6 \mathrm{ml} / \mathrm{min}$. The column and sample temperatures were maintained at $45^{\circ} \mathrm{C}$ and $4^{\circ} \mathrm{C}$, respectively. The gradient program was as follows: linear gradient from $20 \%$ to $60 \%$ B (0-0.5 minutes), isocratic at $60 \%$ B (0.5-1.5 minutes), linear gradient from $60 \%$ to $98 \%$ B (1.50-1.51 minutes), isocratic at $98 \% \mathrm{~B}$ (1.51-2 minutes), linear gradient from $98 \%$ to $20 \%$ B (2-2.01 minutes), and isocratic at 20\% B (2.01-2.5 minutes). All analyses were performed in electrospray ionization positive mode. The mass spectrometer source conditions were as follows: ion spray voltage $+5500 \mathrm{~V}$, source temperature $500^{\circ} \mathrm{C}$, curtain gas 25 psi, GS1 (sheath gas) 30 psi, GS2 (drying gas) 30 psi, collision gas (nitrogen) medium. The compound-specific parameters are outlined in Supplemental Table 1 in which the peak areas of $17 \alpha$ hydroxyprogesterone and DHEA oxime were quantified as the products formed via $17 \alpha$-hydroxylation and the $\mathrm{C} 17,20$-lyase reaction, respectively. For cellular washout experiments, testosterone$2,3,4-{ }^{13} \mathrm{C}_{3}$ was used as the internal standard. Chromatographic peak integration was performed using MultiQuant software (AB Sciex) to obtain the peak area of the analyte expressed as a ratio to the peak area of the internal standard (peak area ratio).

MD Simulations. The X-ray crystal structure of abirateronebound CYP17A1 (Protein Data Bank 3RUK, chain D) was used as the template for MD simulations and PMF calculations. All MD simulations of the protein and the heme prosthetic group were performed using Groningen Machine for Chemical Simulations version 5.1.5 in conjunction with the Groningen Molecular Simulation 54A7 force field (Schmid et al., 2011; Abraham et al., 2015). The simple point charge water model was used to describe the solvent water. Simulations were performed under periodic boundary conditions in a rectangular box. A cutoff of $1.4 \mathrm{~nm}$ was applied to short-range nonbonded interactions, whereas long-range electrostatic interactions were calculated using the particle mesh Ewald algorithm (Darden et al., 1993; Essmann et al., 1995). Abiraterone was predicted to be neutral at pH 7.4 [ChemAxon, (Szegezdi and Csizmadia, 2007)]. Topology parameters for the neutral abiraterone were obtained using the Automated Topology Builder and Repository (Malde et al., 2011).

Abiraterone-bound CYP17A1 was placed in a cubic box of simple point charge water, including neutralizing counterions. A steepest-descents minimization, followed by a position restraint simulation for 100 
picoseconds, was performed under a constant volume (NVT - number of particles, volume and temperature) ensemble. A constant pressure (NPT - number of particles, pressure and temperature) equilibration was performed for 100 picoseconds, using weak coupling to maintain pressure isotropically at 1.0 bar at a temperature of $310 \mathrm{~K}$. A Parrinello-Rahman barostat was used to isotropically regulate pressure, along with a Nosé-Hoover thermostat to maintain temperature, ensuring that a true NPT ensemble was sampled (Parrinello and Rahman, 1981; Nosé, 1984; Hoover, 1985). Production MD simulations were conducted for 250 nanoseconds without any restraints.

PMF Calculations. To calculate the binding free energy of abiraterone, PMF calculations of the abiraterone binding pathway from the CYP17A1 active site were performed along the central axis of the heme using the umbrella sampling method (Torrie and Valleau, 1977; Gordon et al., 2013). The CYP17A1 structure was placed in a rectangular box with dimensions adequate to fulfill the minimum image convention and provide space for "pulling" simulations to occur along the $z$-axis. The chosen reaction coordinate was defined as the axis passing through the heme, normal to the plane of the heme. A harmonic restraint with a force constant of $1000 \mathrm{~kJ} /\left(\mathrm{mol} \cdot \mathrm{nm}^{2}\right)$ was applied to the center of mass $(\mathrm{COM})$ of abiraterone and moved along the reaction coordinate away from the core structure along the $z$-axis over 500 picoseconds with a pull rate of $10 \mathrm{~nm}$ $\mathrm{ns}^{-1}$. The pulling simulation was used to generate a set of 44 reference configurations (windows) separated by $0.05-\mathrm{nm}$ intervals along the reaction coordinate. These windows were then used as starting configurations for umbrella sampling calculations. In each umbrella sampling simulation, the COM of abiraterone was harmonically restrained using a force constant of $1000 \mathrm{~kJ} /\left(\mathrm{mol} \cdot \mathrm{nm}^{2}\right)$ in the $z$-direction to allow sampling of a specific window along the reaction coordinate, where the motion of the abiraterone was not restrained in the $x y$-plane. MD simulations were performed for 20 nanoseconds with each window, providing a total simulation time of 880 nanoseconds for umbrella sampling.

The PMF profile was constructed using the weighted histogram analysis method (Kumar et al., 1992; Hub et al., 2010). The first 4 nanoseconds of each window were taken as equilibration and were therefore not included in the calculation of PMF values. The $K_{d}$ was calculated from the PMF, W(z), using eq. 5 (Chen and Chung, 2012), where $\pi \mathrm{r}^{2}$ is the cross-sectional area of the binding path, $N_{A}$ is Avogadro's number, and $z_{\min }$ and $z_{\max }$ are the $z$-positions of the abiraterone COM when fully bound to CYP17A1 and when in the bulk solvent, respectively. The radius $R$ of the binding path was estimated as $0.5 \mathrm{~nm}$ based on the size of the heme porphyrin moiety.

$$
K_{d}^{-1}=1000 \pi r^{2} N_{A} \int_{Z \min }^{Z \max } \exp [-\mathrm{W}(\mathrm{z}) / \mathrm{kT}] \mathrm{dz}
$$

$\Delta G$ for the binding of abiraterone to CYP17A1 was calculated using eq. 6 , where $\Delta G$ is the binding free energy, $R$ is the universal gas constant, $T$ is the temperature, and $K_{d}$ is the equilibrium dissociation constant.

$$
\Delta G=-R T \ln K_{d}
$$

The volume of the binding pocket was estimated using the mdpocket program (Schmidtke et al., 2011). The pocket volume calculations were performed in an unliganded trajectory of CYP17A1-abiraterone simulation to estimate fluctuations in the presence of a bound ligand.

Development of a Mechanistic PK-PD Model Linking Abiraterone PK and CYP17A1-Abiraterone Binding Kinetics to Steroidogenic Flux. We also sought to understand CYP17A1 target occupancy by abiraterone in the context of its in vivo PK (Dahl and Akerud, 2013). Considering the two-step slow-binding inhibition mechanism as shown in Fig. 1B, time-dependent changes in the concentration of each enzyme species (i.e., $[E],[E I],\left[E I^{*}\right]$ ) were simulated after a single 1000- or 500-mg dose of AA (Acharya et al., 2012). Dynamic in vivo target occupancy was determined by the summation of $[E I]$ and $\left[E I^{*}\right]$.

Equations 7, a-d describe the system of differential equations that was consecutively solved in parallel over very small intervals to simulate in vivo target occupancy (Vauquelin, 2017). The initial value of $[E]$ was defined as the steady-state concentration of CYP17A1 in vivo. CYP17A1 has an abundance of approximately $35,000 \mathrm{pmol} / \mathrm{g}$ tissue. Considering the weight ( $10 \mathrm{~g})$ and volume of the adrenal glands $(0.0085 \mathrm{l})$ gives a steadystate concentration of $41 \mu \mathrm{M}$. The flux in the concentration of free inhibitor $[I]$ near the target was assumed to be equal to the plasma concentration-time profile of abiraterone after AA administration.

$$
\begin{gathered}
\frac{d[E]}{d t}=k_{\text {syn }}-k_{\text {deg }}[E]+k_{2}[E I]-k_{1}[E][I] \\
\frac{d[E I]}{d t}=k_{1}[E][I]-k_{2}[E I]+k_{4}\left[E I^{*}\right]-k_{3}[E I] \\
\frac{d\left[E I^{*}\right]}{d t}=k_{3}[E I]-k_{4}\left[E I^{*}\right] \\
\text { Occupancy }=[E I]+\left[E I^{*}\right]
\end{gathered}
$$

Supplemental Table 2 presents a summary of the relevant input parameters. Values for microscopic rate constants $k_{3}$ and $k_{4}$ were derived from the preincubation time-dependent kinetic experiments. As highlighted in eq. 8a, the overall target affinity $\left(K_{i}^{*}\right)$ of abiraterone for CYP17A1 can be described as a composite of the various microscopic rate constants. With rearrangement, eq. $8 \mathrm{~b}$ emphasizes the equivalence of $\left(k_{3}+k_{4}\right) / k_{2}$ and the $k_{4} /\left(k_{1} \cdot K_{i}^{*}\right)$ ratios (Vauquelin, 2017).

$$
\begin{gathered}
K_{i}^{*}=\frac{k_{2} k_{4}}{k_{1}\left(k_{3}+k_{4}\right)} \\
\frac{k_{3}+k_{4}}{k_{2}}=\frac{k_{4}}{k_{1} K_{i}^{*}}
\end{gathered}
$$

For induced-fit binding, slow dissociation and high affinity rely on the stability of the $\left[E I^{*}\right]$, a scenario necessitating that $k_{4}$ becomes the kinetic bottleneck of the dissociation process (Copeland et al., 2006; Copeland, 2013; Vauquelin, 2017). Hence, to qualify as an induced-fit binder, it follows that $\left(k_{3}+k_{4}\right) \ll k_{2}$ and $k_{4} \ll\left(k_{1} K_{i}^{*}\right)$ (Vauquelin, 2017). Consequently, the eventual estimates of $k_{1}$ and $k_{2}$ satisfied these inequality relationships as well as the $K_{i, a p p}$ of the initial $[E I]$ complex obtained from kinetic analyses.

In subsequent PK-PD simulations, CYP17A1 engagement varying as a function of both abiraterone concentration and time was prospectively correlated with the extent of DHEA-sulfate suppression in a dynamic in vitro model of intracellular steroidogenesis as developed by Ahmed et al. (Ahmed et al., 2018) (Supplemental Methods). All analyses were performed in RStudio 1.1.456 using the deSolve package (R Core Team, 2013).

Correlating Abiraterone PK with Androgen Reduction. A phase II trial by Szmulewitz et al. (2018) demonstrated comparable efficacy of low-dose AA ( $250 \mathrm{mg}$ with a low-fat meal) to standard-dose AA (1000 mg fasting). Utilizing plasma abiraterone measurements collected after 8 days of therapy (before and 2,3, and 4 hours after dosing) in the low-dose and high-dose groups, differences in PK parameters [i.e., area under the plasma concentration-time curve $\left(\mathrm{AUC}_{0-4}\right.$ hours $)$ and peak plasma concentrations $\left(C_{\max }\right)$ ] were evaluated and compared with AUC and $C_{\max }$ values derived from two phase I trials (O'Donnell et al., 2004; Attard et al., 2008) studying the 500-mg dose of AA (fasted) in castrate patient cohorts. Group comparisons of log-transformed PK data were performed via one-way analysis of variance, followed by Dunnett's multiple comparison test. Mean AUC and $C_{\max }$ ratios as well as 95\% confidence intervals (CIs) for pairwise comparisons were calculated by retransformation of the logarithmic results.

\section{Results}

Abiraterone and D4A Cause Slow- and Tight-Binding Inhibition of CYP17A1. The binding of abiraterone and D4A to CYP17A1 was characterized by monitoring the formation of both $17 \alpha$-hydroxyprogesterone and DHEA oxime as a function of preincubation time. Semilogarithmic plots 
A

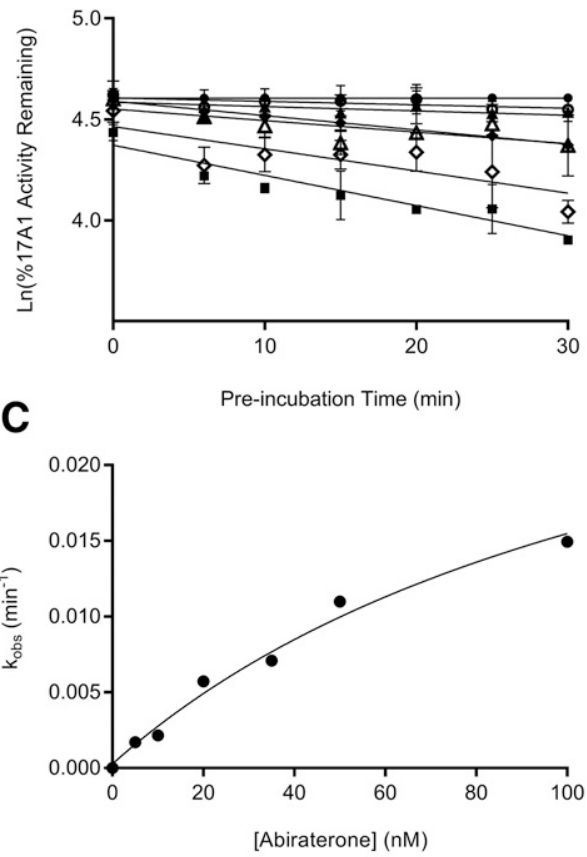

E

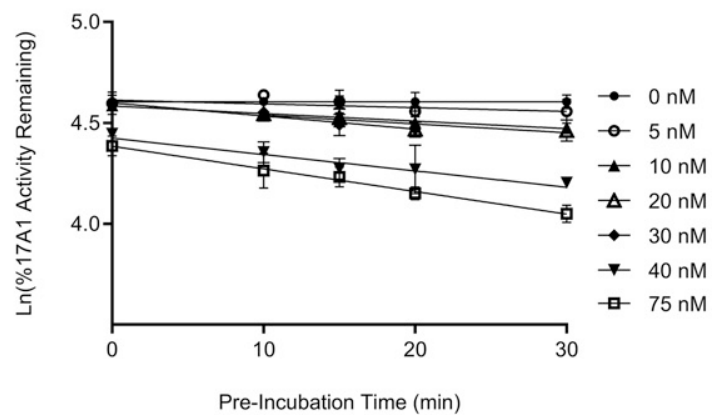

G

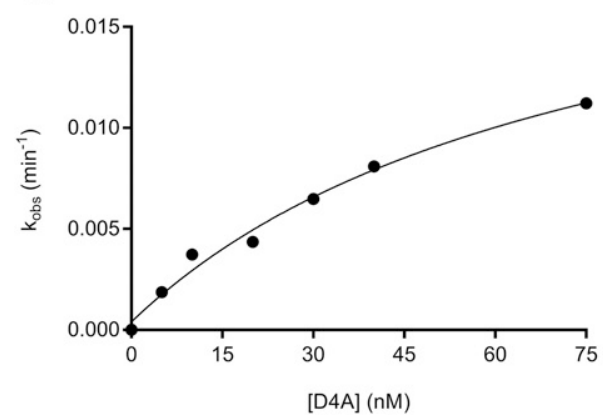

B

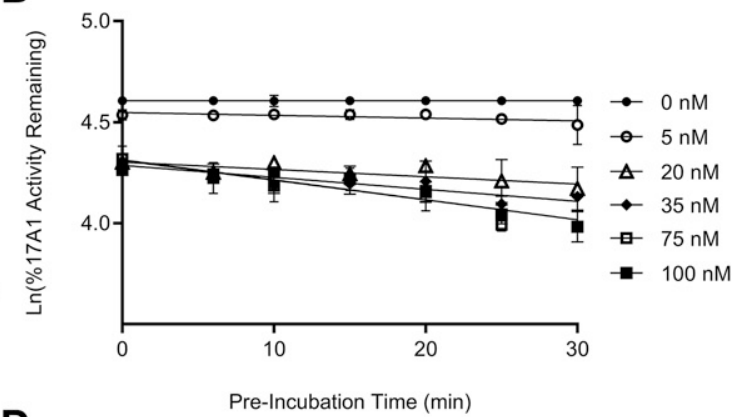

D

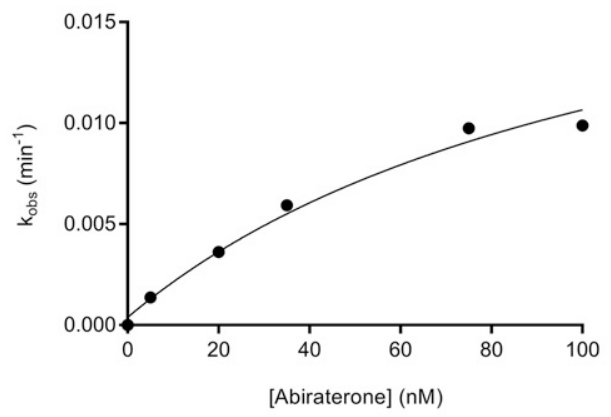

F

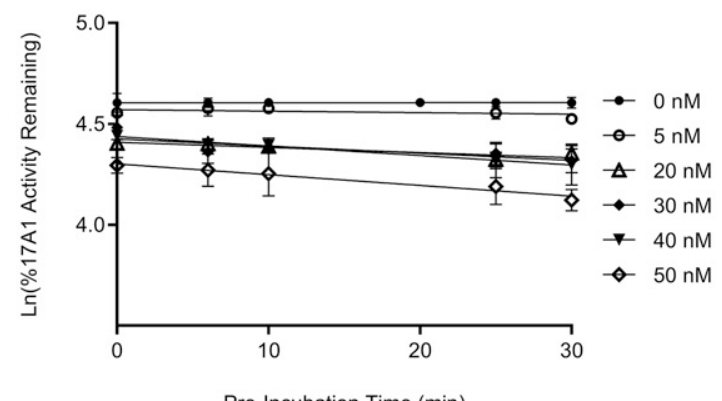

H

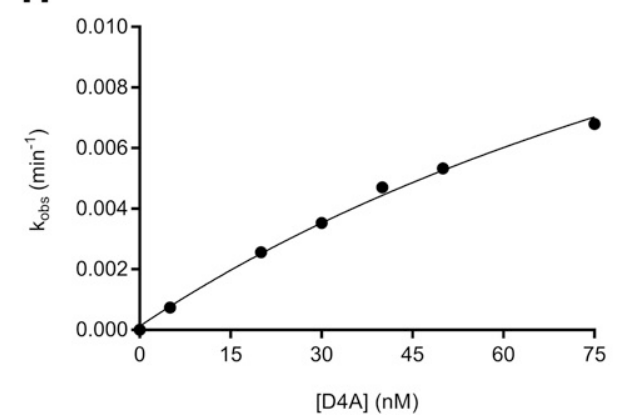

Fig. 2. Preincubation time-dependent inhibition of CYP17A1 by abiraterone and D4A. Semilogarithmic plots illustrate time- and concentrationdependent inhibition of CYP17A1 by (A and B) abiraterone or ( $\mathrm{E}$ and $\mathrm{F}$ ) D4A using progesterone and $17 \alpha$-hydroxypregnenolone as probe substrates for $17 \alpha$-hydroxyprogesterone and dehydroepiandrosterone formation, respectively. The relationship between the observed first-order rate constants for the onset of inhibition $\left(k_{o b s}\right)$ values determined from $(\mathrm{A}, \mathrm{B}, \mathrm{E}$, and $\mathrm{F})$ and inhibitor concentration was further investigated in $(\mathrm{C}, \mathrm{D}, \mathrm{G}$, and $\mathrm{H})$, and data were subsequently fitted to eq. 3a to calculate the forward $\left(k_{3}\right)$ and reverse $\left(k_{4}\right)$ isomerization constants via nonlinear regression analysis. Goodness of fits $\left(\mathrm{R}^{2}\right)$ for $(\mathrm{C}, \mathrm{D}, \mathrm{G}$, and $\mathrm{H})$ were determined to be $0.984,0.984,0.986$, and 0.996 , respectively. Each point in the semilogarithmic plots represents the mean \pm S.D. of triplicate determinations.

illustrated time- and concentration-dependent decreases in residual CYP17A1 activity (relative to a $[\mathrm{I}]=0$ preincubation control sample) and enabled determination of the first-order rate constants for onset of inhibition $\left(k_{o b s}\right)$ at various concentrations of abiraterone (Fig. 2, A and B) and D4A (Fig. 2, E and
F) using eq. 1 . The hyperbolic dependence of $k_{o b s}$ on abiraterone/D4A concentration as observed in the kinetic plots (Fig. 2, $\mathrm{C}, \mathrm{D}, \mathrm{G}$, and $\mathrm{H}$ ) is indicative of a two-step slow-binding inhibition mechanism (Fig. 1B) (Morrison and Walsh, 1988; Copeland, 2000). Concentration-dependent reduction of 
CYP17A1 activity in the absence of preincubation ( $y$-intercept values in Fig. 2, A, B, E, and F) further substantiates a twostep model, in which the rapid, reversible binding of $E+I$ to form $E I$ is manifested as an instantaneous effect on the initial reaction velocity $\left(v_{i}\right)$. The apparent inhibition constant for the initial $E I$ complex $\left(K_{i}\right.$ app) was determined by fitting the dependence of fractional velocity $\left(v_{i} / v_{0}\right)$ on inhibitor concentration using eq. 2 (Fig. 3, A, B, E, and F; Table 1). In the second step, the binding of abiraterone/D4A to CYP17A1 subsequently induces a time-dependent conformational change in CYP17A1 that results in a high-affinity $E I^{*}$ complex (Fig. 1B). Nonlinear regression analyses based on eq. 3a allowed the forward $\left(k_{3}\right)$ and reverse $\left(k_{4}\right)$ isomerization constants to be established (Table 1).

The Tight-Inhibited Complex (EI*) Demonstrates Significant Increases in Inhibitory Potency. Reversible inhibition of CYP17A1 by abiraterone (Fig. 3, C and D) and D4A (Fig. 3, G and $\mathrm{H}$ ) was assessed. The intersection of lines above the $x$-axis in double reciprocal Lineweaver-Burk transformations revealed mixed-mode inhibition of CYP17A1 by both abiraterone (Supplemental Fig. 4, A and B) and D4A (Supplemental Fig. 4, C and D). Hence, the $K_{i}$ app values determined for abiraterone and D4A were corrected to the true $K_{i}$ (Table 1 ). With acquisition of $k_{3}, k_{4}$, and $K_{i}$, the overall inhibition constants for the final $E I^{*}$ complex $\left(K_{i}^{*}\right)$ were calculated using eq. 4 . For abiraterone and $\mathrm{D} 4 \mathrm{~A}$, the isomerization of $E I$ to $E I^{*}$ was accompanied by significant increases in inhibitory potencies $\left(K_{i}\right.$ to $K_{i}^{*}$ in Table 1), expounding the thermodynamic stabilization of the ground state of $E I^{*}$ versus $E I$ (Tonge, 2018). Using eq. 3b, which assumes that $k_{4}$ is rate-determining, both abiraterone and D4A demonstrated prolonged residence time within the binding domain of CYP17A1 (Table 1). However, as reflected in Table 1, despite comparable inhibitory potencies across both $17 \alpha$-hydroxylase and C17,20-lyase reactions for abiraterone and D4A, D4A exhibited longer residence time on CYP17A1 when the C17,20-lyase reaction was measured (95 hours vs. 29-42 hours), highlighting the discordance between affinity and residence time measurements.

Binding Free Energy of the CYP17A1-Abiraterone Complex is Consistent with Kinetic Analyses. Given that the X-ray crystal structure reported by DeVore and Scott (2012) has abiraterone bound to CYP17A1, subsequent MD simulations considered the CYP17A1-abiraterone interaction only. The potential of mean force (PMF) profile was generated to predict the binding affinity of abiraterone to CYP17A1. In the reported binding mode (referred to hereafter as pose A), the abiraterone steroid moiety orients near perpendicular to the plane of the heme. Based on the PMF profile of abiraterone unbinding pathway, a distinct energy well occurs at the reaction coordinate, $z=5 \AA$ (Fig. 4A). The PMF calculations converged after 4 nanoseconds; further extension of simulations to 20 nanoseconds for each umbrella window did not appreciably change the PMF profile or binding free energy ( $\mathrm{SD}=0.5 \mathrm{kcal} / \mathrm{mol})$. The binding mode associated with the energy well is essentially identical to that observed in the X-ray crystal structure, in which the $\mathrm{N}$ atom of abiraterone forms a coordinate bond with heme Fe $(<2.6 \AA)$. The computationally determined free energy of pose A was $-14.5 \mathrm{kcal} / \mathrm{mol}$ and compares well to the experimental in vitro estimates $(-12.5$ and -12.8 $\mathrm{kcal} / \mathrm{mol}$ for $K_{i}^{*}=0.42$ and $0.39 \mathrm{nM}$ depending on substrate, Table 1). Consistent with the PMF profile, only one abiraterone binding pose $\mathrm{A}$ is identified using this approach (Fig. 4B).

Prolonged CYP17A1 Binding of Abiraterone Was Confirmed in Cellular Washout Experiments. To ensure that any kinetic selectivity observed at the level of the purified recombinant CYP17A1 target could be qualitatively recapitulated in complex biologic systems, cellular washout experiments utilizing human prostate cancer cells (VCaP) were performed. Formation of DHEA at various concentrations of $17 \alpha$-hydroxypregnenolone exhibited Michaelis-Menten kinetics, establishing the cellular functionality of CYP17A1 (Fig. 5A). In coincubation experiments, both abiraterone (20 $\mu \mathrm{M})$ and ketoconazole $(25 \mu \mathrm{M})$ produced depressions of the maximal response observed after 2 hours (quantified via DHEA formation in vehicle controls) (Fig. 5B), demonstrating inhibition of C17,20-lyase reaction by abiraterone and ketoconazole. Upon preincubation of abiraterone and subsequent washout, continued suppression of CYP17A1 activity was observed up to 60 minutes (Fig. 5C). In contrast, minimal prolongation of DHEA suppression was observed after washout of known reversible CYP17A1 inhibitor ketoconazole (Fig. 5C).

Identification of a Second Binding Mode Confirms the Two-Step Induced-Fit Binding Mechanism Observed in Kinetic Experiments. In addition to the tightbinding mode, the kinetic experiments performed here identified a fast, reversible binding component of abiraterone to CYP17A1. Beyond the PMF calculations performed over a short simulation timescale, a long, unrestrained MD simulation (250 nanoseconds) was performed to explore additional binding event(s). MD simulations of the abiraterone-CYP17A1 complex demonstrated two dominant binding modes of abiraterone within the active site. In pose $A$, the steroid moiety of abiraterone is oriented near vertically above the plane of the heme between the F- and G- helices. This is similar to the chemical interactions and orientation reported in the X-ray crystal structure (DeVore and Scott, 2012) and pose A observed in the PMF calculations (Fig. 4B).

In the second binding mode, referred to as pose $B$, the plane of the steroid moiety is orientated parallel to the plane of the heme. The $\alpha$-face of the steroid nucleus packs almost entirely along the I-helix, whereas in pose A, only a portion of the structure packs against the I-helix (Fig. 4C). In pose $\mathrm{A}$, $\mathrm{H}$-bonding occurs between the $3 \beta-\mathrm{OH}$ group of abiraterone and Asn202. In pose B, H-bonding occurs between the $3 \beta-\mathrm{OH}$ group and Arg 239 (2.2 $\AA$ ) and Asp 298 (2.4 $\AA$ ) of the G- and I-helices, respectively. We propose that the binding mode of abiraterone identified in pose B may represent the fast, reversible interaction that occurs prior to heme coordination.

Simulated Target Occupancy after a Single In Vivo Dose of Abiraterone Exceeds Its PK Half-Life. Simulations of apparent CYP17A1 occupancy after a single 1000- or 500-mg dose of AA were performed using numerical integration over a system of differential equations for the two-step slow-binding enzyme inhibition mechanism (Vauquelin, 2017). The terminal elimination $t_{1 / 2}$ of abiraterone is approximately 16 hours (Fig. 6A). At the end of the dosing interval of 24 hours, when most abiraterone has been eliminated systemically, an on-target peak-to-trough occupancy ratio (in which trough is measured 24 hours after dosing) was calculated to be 1.1 for both 1000 - and 500-mg doses, indicating approximately constant apparent target occupancy between two consecutive doses (Vauquelin, 
A

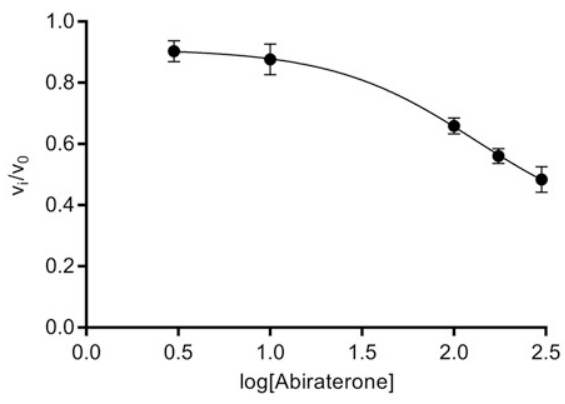

C

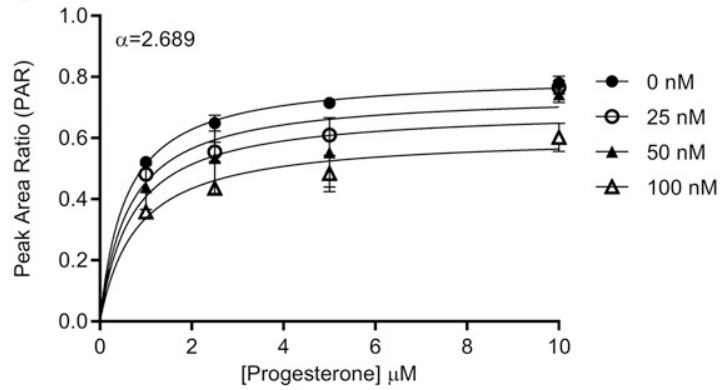

E

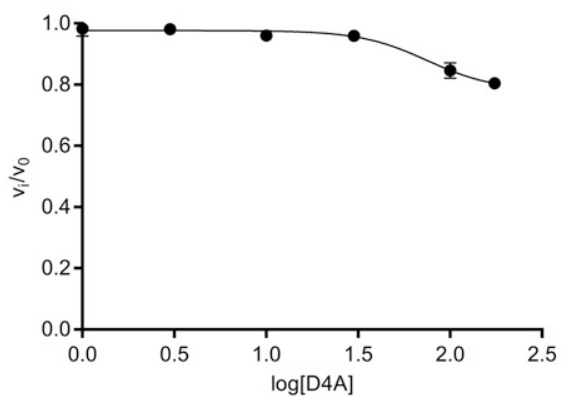

G

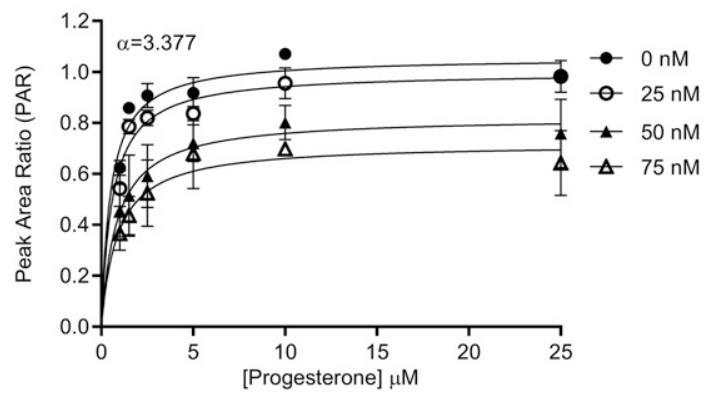

B

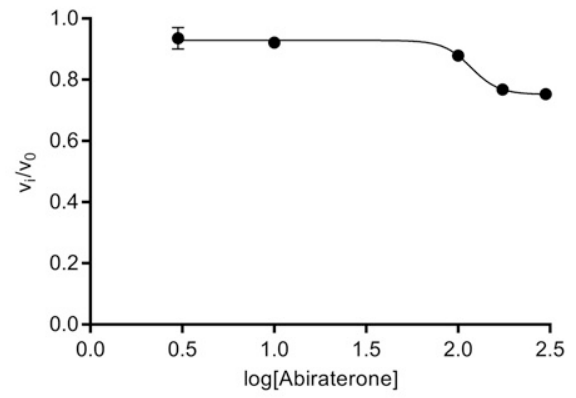

D

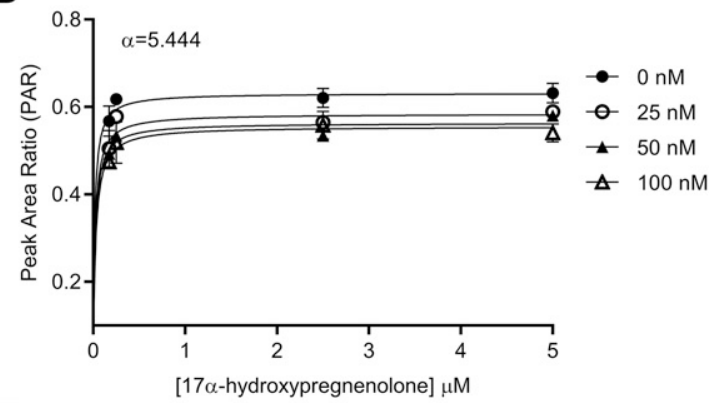

F

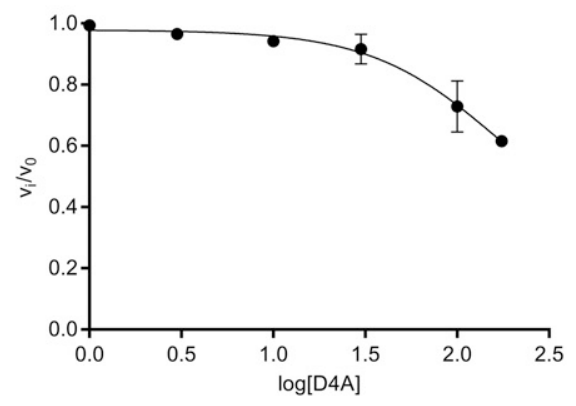

H

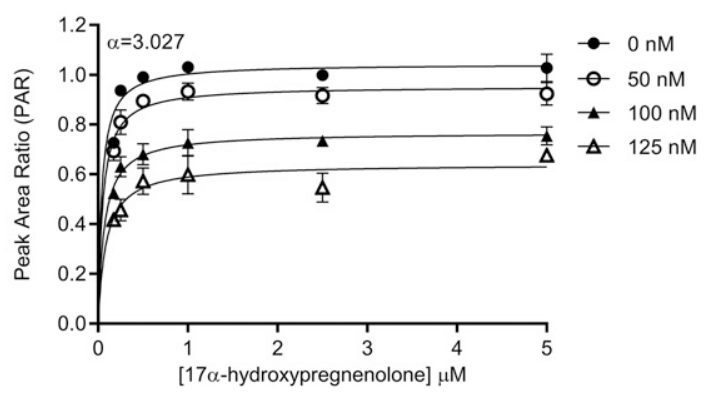

Fig. 3. Reversible inhibition of CYP17A1 by abiraterone and D4A. Concentration-response plots represent the initial inhibited state (EI) of the CYP17A1-mediated $17 \alpha$-hydroxylase and C17,20-lyase enzymatic reactions when slow-, tight-binding inhibitors (A and B) abiraterone and (E and F) D4A were introduced. The measured initial velocities $\left(v_{i}\right)$ were used together with velocity of the uninhibited reaction $\left(v_{0}\right)$ to calculate the fractional velocities across various concentrations of abiraterone and D4A (1-300 nM). Using eq. 2, apparent inhibition constants $\left(K_{i}, a p p\right)$ for the initial CYP17A1-inhibitor encounter complexes were first obtained from the midpoint of isotherm curves. $K_{i}$, app values were subsequently used in nonlinear regression analyses (eq. 3a) to determine the forward $\left(k_{3}\right)$ and reverse $\left(k_{4}\right)$ isomerization constants. Reversible inhibition experiments were also performed in the presence of multiple substrate (progesterone or $17 \alpha$-hydroxypregnenolone) and (C and D) abiraterone or ( $\mathrm{G}$ and H) D4A concentrations. To discern the mode of inhibition, Michaelis-Menten plots generated were subjected to double reciprocal transformations to yield Lineweaver-Burk graphs as presented in Supplemental Fig. 4. Based on the identified mode of inhibition, the inhibition constant for the initial EI complex $\left(K_{i}\right)$ could eventually be derived from $K_{i}$, app. Each point represents the mean \pm S.D. of triplicate determinations. 
TABLE 1

Summary of kinetic constants and dissociation $t_{1 / 2}$ for the inhibition of CYP17A1 by abiraterone and D4A

\begin{tabular}{lccccccc}
\hline Inhibitor & CYP17A1 Activity & $\boldsymbol{K}_{\boldsymbol{i}, \boldsymbol{a p p}}(\mathrm{nM})^{a}$ & $\boldsymbol{k}_{3}\left(\mathrm{~min}^{-1}\right)^{b}$ & $\boldsymbol{k}_{4}\left(\mathrm{~min}^{-1}\right)^{b}$ & $\boldsymbol{K}_{\boldsymbol{i}}(\mathrm{nM})^{\boldsymbol{c}}$ & $\boldsymbol{K}_{\boldsymbol{i}}^{*}(\mathrm{nM})$ & Dissociation $t_{1 / 2}(\mathrm{~h})$ \\
\hline Abiraterone & $17 \alpha$-Hydroxylase & $132 \pm 2.02$ & 0.0353 & 0.000275 & 50.7 & 0.39 & 42.0 \\
& C17,20-Lyase & $118 \pm 1.13$ & 0.0224 & 0.000384 & 24.8 & 0.42 & 30.1 \\
D4A & $17 \alpha$-Hydroxylase & $76 \pm 1.21$ & 0.0218 & 0.000396 & 23.5 & 0.42 & 29.2 \\
& C17,20-Lyase & $163 \pm 3.02$ & 0.0219 & 0.000121 & 57.4 & 0.32 & 95.5 \\
\hline
\end{tabular}

${ }^{a}$ From plot of $\frac{\mathrm{v}_{\mathrm{i}}}{\mathrm{V}_{0}} \mathrm{vs}$. [I]. Data represented as means \pm S.D. of triplicate determinations.

${ }^{b}$ From plot of $k_{o b s}$ vs. [I].

${ }^{c}$ Value of $K_{i, a p p}$ corrected for mixed-mode inhibition.

2017). Notably, $>80 \%$ apparent CYP17A1 engagement was preserved despite a dose reduction to $500 \mathrm{mg}$.

Translation of CYP17A1 Occupancy to PD Outcomes Rationalized a Clinically Observed PK-PD Disconnect. Nevertheless, the translation of target occupancy to efficacy outcomes requires additional consideration of the amount of target that must be complexed with abiraterone to elicit the desired PD effect (i.e., target vulnerability). When multiple dosing of AA for 72 hours was used to perturb a baseline model of steroidogenesis, the model predicted comparable attenuations in intracellular DHEA-sulfate concentrations for the $1000-$ and $500-\mathrm{mg}$ doses, calculated to be $3.26 \%$ and $4.68 \%$ at 72 hours of baseline measurements (without inhibition), respectively (Fig. $6 \mathrm{~B}$ ).

This PK-PD disconnect paralleled reported clinical findings from a phase II trial comparing low-dose AA $(250 \mathrm{mg}$ daily with a low-fat meal) versus standard-dose AA (1000 mg daily fasting). The systemic exposure ( $\mathrm{AUC}_{0-4}$ hours $)$ and $C_{\max }$ of abiraterone were significantly higher in the standard-dose arm compared with the low-dose group (Fig. 7) (mean $\mathrm{AUC}_{0-4}$ hours ratio $=2.08,95 \%$ CI: $1.02-4.24$; mean $C_{\max }$ ratio $=2.02$, 95\% CI: 1.01-4.05). Nevertheless, equipotent reduction of plasma DHEA-S levels was observed (Szmulewitz et al., 2018), confirming that the clinical efficacy of CYP17A1 inhibition was maintained even at lower systemic exposure of abiraterone.

Notably, as presented in Fig. 7B, comparable $C_{\max }$ measurements (mean $C_{\max }$ ratio $=0.98,95 \% \mathrm{CI}=0.42-2.26$ ) were observed between a 500-mg dose of AA administered under fasting conditions and $250 \mathrm{mg}$ of AA administered with food. The mean AUC ( $\mathrm{AUC}_{0-12}$ hours and $\mathrm{AUC}_{0-72}$ hours $)$ associated with the 500-mg dose was expectedly greater than $\mathrm{AUC}_{0-4}$ hours, associated with the low-dose treatment arm (mean AUC ratio $=2.83,95 \% \mathrm{CI}=1.42-5.62)($ Fig. $7 \mathrm{~A})$. Based on PK measurements made up to 72 hours in a phase I dose escalation trial (Acharya et al., 2012), the calculated $\mathrm{AUC}_{0-4}$ hours is approximately $50 \%$ of the total AUC across different doses. Taken together, a 500-mg dose of AA is expected to yield similar exposure to $250 \mathrm{mg}$ of AA administered with food, representing a viable alternative to utilizing the food effect to enhance the systemic exposure of abiraterone.

\section{Discussion}

The translational disconnect between preclinical and clinical pharmacology is a perennial challenge. Incorrect assumptions underlying various processes in the causal chain between drug exposure and pharmacological response often restrict the success of in vitro-to-in vivo extrapolation techniques. By elucidating the interaction between CYP17A1 and abiraterone/D4A, we demonstrate how inadequate characterization of target association-dissociation kinetics precludes an accurate determination of the in vivo time course of drug action.

For the first time, our results chronicle the profiling of abiraterone and its active metabolite D4A as slow-, tightbinding inhibitors of steroidogenic CYP17A1 adhering to a two-step induced-fit mechanism. As highlighted in Table 1, the conversion from the initial EI complex to the final EI* was accompanied by significant ( $>50$-fold) increases in inhibitory potencies $\left(K_{i}\right.$ to $\left.K_{i}^{*}\right)$ for both CYP17A1-mediated reactions. Hence, our results underscore how functional comparisons of compound inhibitory activity with the conventional assumption of rapid equilibrium may hinder detection of the timedependent effects that are essential for holistic assessment of the true inhibitory potencies of nonclassic inhibitors. Notably, the $K_{i}$ and $K_{i}^{*}$ values obtained largely reconcile the considerable incongruity observed in previous studies examining the nature and potency of CYP17A1 inhibition by abiraterone and D4A. $K_{i}$ values quantifying the initial mixed-mode inhibition of progesterone $17 \alpha$-hydroxylation by abiraterone $(50.7 \mathrm{nM})$ and $\mathrm{D} 4 \mathrm{~A}(23.4 \mathrm{nM})$ are comparable to those obtained by Garrido et al. (2014) $\left(K_{i}=27 \mathrm{nM}\right.$ for abiraterone and $22 \mathrm{nM}$ for D4A). Conversely, spectral ligand binding assays monitoring time- and concentration-dependent heme coordination have implicated abiraterone and D4A as tight-binding inhibitors with extremely high affinity for CYP17A1 (Garrido et al., 2014). This alternative approach measures the dissociation of I from $E I^{*}\left(K_{d}=2.6 \mathrm{nM}\right.$ for abiraterone and $<1 \mathrm{nM}$ for D4A) and is analogous to the $K_{i}^{*}$ established in the present work (Table 1). Independently, PMF calculations from MD simulations yielded binding affinity estimates of abiraterone with CYP17A1 ( $-14.5 \mathrm{kcal} / \mathrm{mol})$ that also verified the experimentally measured binding affinity $(-12.5$ and $-12.8 \mathrm{kcal} / \mathrm{mol}$ for $K_{i}^{*}=0.42$ and $0.39 \mathrm{nM}$ ). The convergence of results from MD simulations and biochemical analyses confirms the formation of the $E I^{*}$ complex between abiraterone and CYP17A1.

Our kinetic experiments have confirmed that the highaffinity interactions of abiraterone/D4A with CYP17A1 can be attributed to a two-step induced-fit mechanism. However, the conservation of the global conformation of CYP17A1 in the presence of abiraterone (Supplemental Fig. 5, A and B), D4A (Supplemental Fig. 5, C and D), and various ligands (Supplemental Fig. 6) (DeVore and Scott, 2012; Petrunak et al., 2014, 2017) suggest that abiraterone or D4A can bind in the active site in the same orientation as that observed in the X-ray crystal structure, which paradoxically renders the proposed induced-fit mechanism untenable. Nevertheless, molecular docking simulations are static by nature and are unable to simulate protein flexibility throughout binding (Salmaso and Moro, 2018). Indeed, cytochrome P450 enzymes with large binding sites have been demonstrated to undergo dramatic 
A

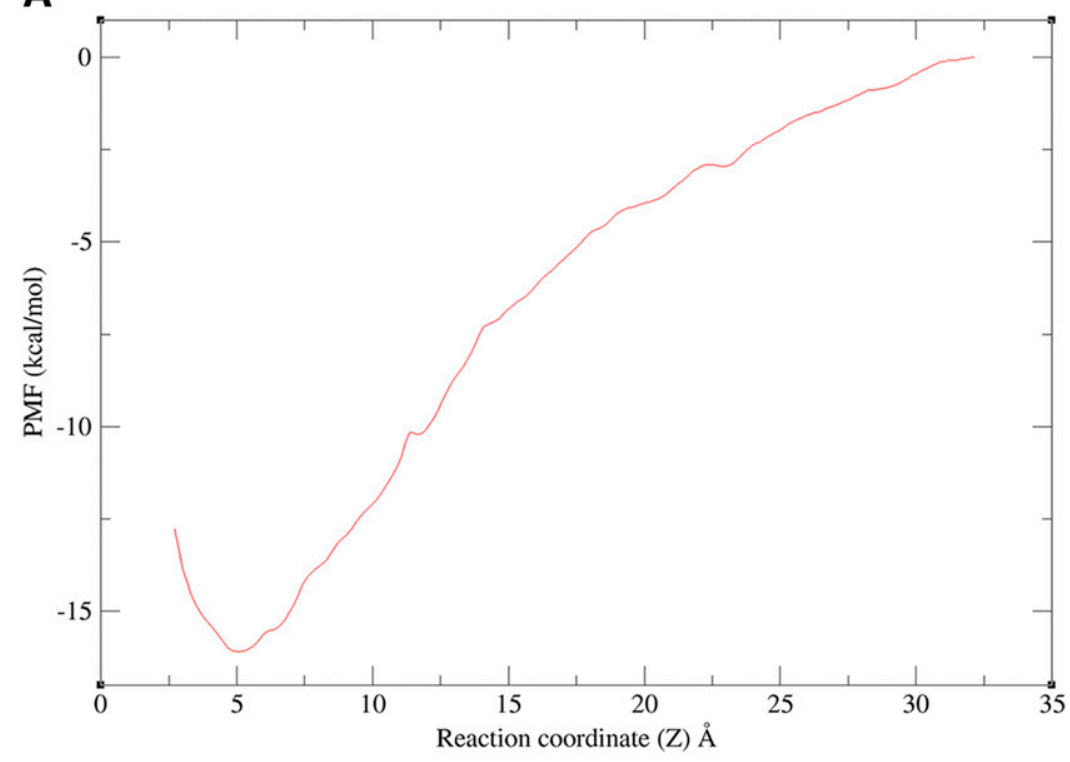

B

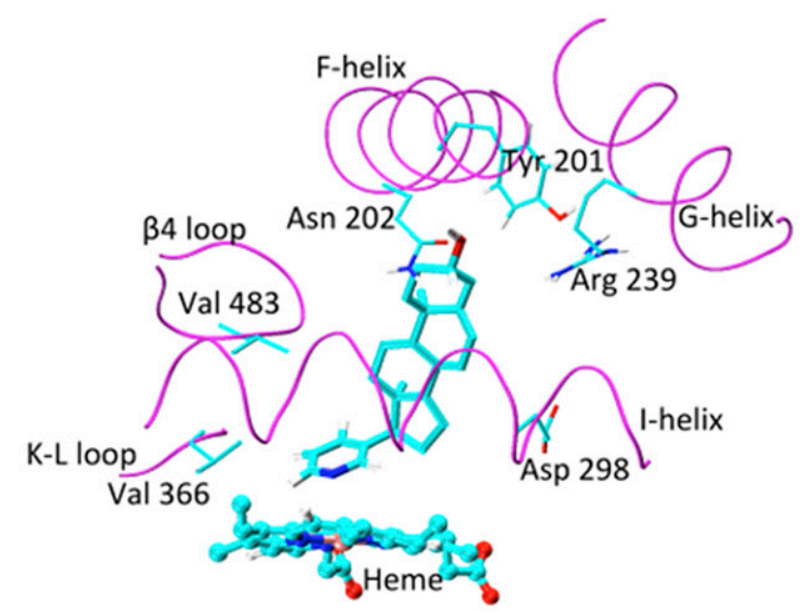

C

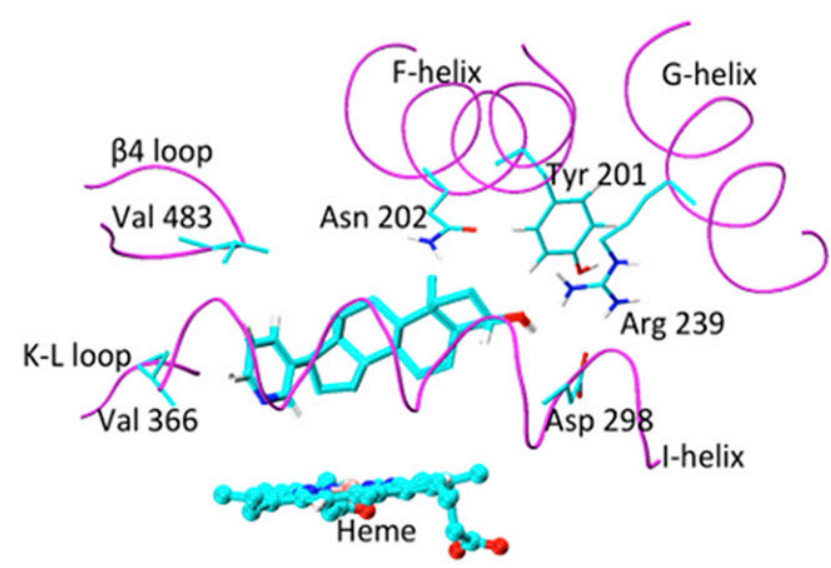

Fig. 4. $M D$ simulations investigating the binding of abiraterone to CYP17A1. (A) PMF profile for the unbinding of abiraterone from CYP17A1. (B) Long, unrestrained MD simulations demonstrated two dominant binding modes of abiraterone in CYP17A1 catalytic site. In pose A, the plane of the steroid moiety is near perpendicular to the plane of the heme. (C) In pose B, abiraterone is parallel to the plane of the heme. The key-binding region of the CYP17A1 active site is shown in magenta. The side chain atoms of important binding residues are shown as sticks, abiraterone as thick sticks, and heme as ball and sticks. C, O, and $\mathrm{N}$ atoms are shown in cyan, red, and blue, respectively. conformational changes to accommodate and optimize the binding of ligands with diverse structures and shapes (Ekroos and Sjogren, 2006; Nair et al., 2016; Sevrioukova and Poulos, 2017).

MD simulations demonstrated that the pyridine ring of abiraterone occupies an unfilled space of CYP17A1 between
Val366 (on a K-L loop) and Val483 ( $\beta 4$ loop) (DeVore and Scott, 2012), where its steroid nucleus binds near parallel to the plane of heme (pose B in Fig. 4C). This parallel pose has been comparatively observed for steroidal ligands binding to CYP19A1 [e.g., Protein Data Bank 3EQM, 5JKW; (Ghosh et al., 2018)]. 


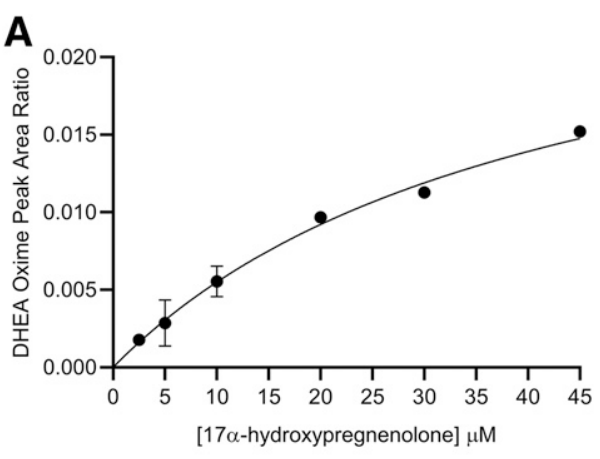

B
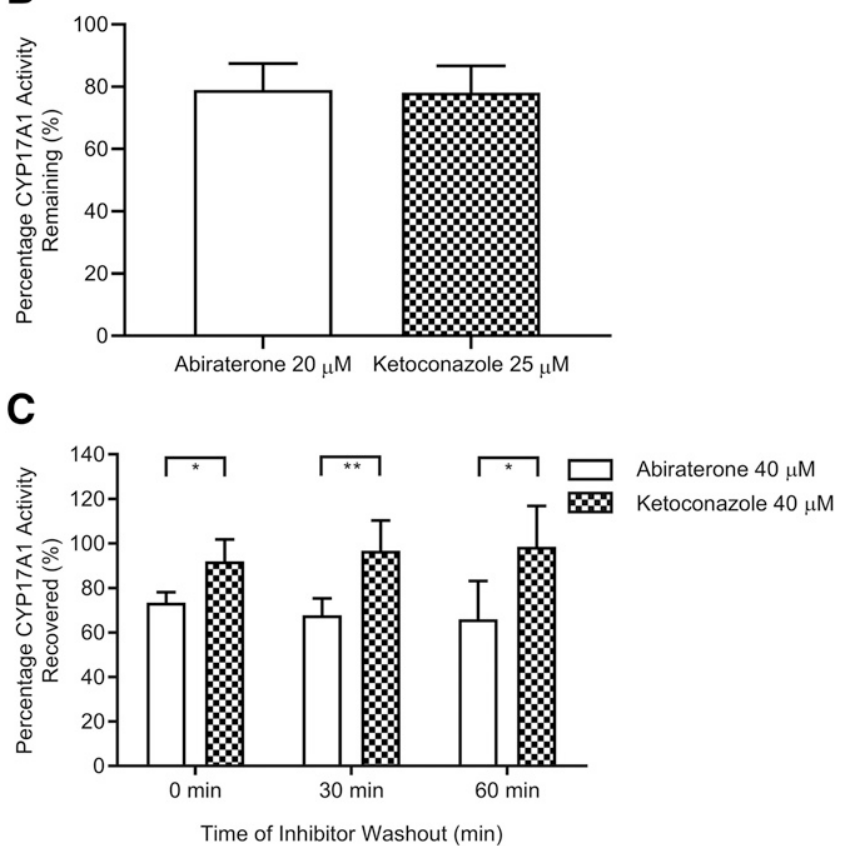

Fig. 5. VCaP cell-based washout experiments to assess the phenotypic consequence of CYP17A1 engagement after inhibitor removal. (A) DHEA formation at increasing concentrations of $17 \alpha$-hydroxypregnenolone conformed to saturable Michaelis-Menten kinetics $\left(R^{2}=0.974\right)(\mathrm{B})$ The effect of either abiraterone $(20 \mu \mathrm{M})$ or ketoconazole $(25 \mu \mathrm{M})$ coincubation on $17 \alpha$-hydroxypregnenolone-mediated DHEA formation in VCaP cells. Cells were incubated for 2 hours at $37^{\circ} \mathrm{C}$. (C) Time dependent reversal of CYP17A1 inhibition by abiraterone or ketoconazole as quantified via restoration of DHEA production. $\mathrm{VCaP}$ cells were preincubated for 2 hours without (control) or with abiraterone $(40 \mu \mathrm{M})$ or ketoconazole $(40 \mu \mathrm{M})$, washed, and further incubated with fresh medium for the indicated washout periods before final incubation with $30 \mu \mathrm{M} 17 \alpha$-hydroxypregnenolone for 30 minutes. DHEA oxime formation, representing residual CYP17A1 activity in (B) and recovery of CYP17A1 activity in (C) was expressed as the percentage of maximal DHEA oxime produced in the corresponding vehicle controls. In (C), an unpaired $t$ test revealed overall significant difference between treatment arms across the 60-minute washout period ( 0 minutes: one-sided $P$ value $=0.0158 ; 30$ minutes: onesided $P$ value $=0.0071 ; 60$ minutes: one-sided $P$ value $=0.0281)$. Data in (A) represent the means \pm S.D. from two experiments with triplicate determinations, whereas data in $(\mathrm{B}$ and $\mathrm{C})$ represent the means \pm S.D. from at least three experiments with triplicate determinations. ${ }^{*} P<0.05$; $* * P<0.01$

To probe the molecular basis of the two binding poses A and $\mathrm{B}$, we investigated and established conformational changes in the F-helix (Supplemental Table 3) and the region connecting the F- and G-helices (Nair et al., 2016), which are consistent with the secondary X-ray crystal structure of abirateronebound CYP17A1 (Supplemental Fig. 7). Our MD simulations further demonstrated fluctuations of ligand-bound CYP17A1 active site volume (496-1023 $\AA^{3}$ ) with flexibilities noted for the F-helix, K-L loop, and $\beta 4$ loop region. Taken together, these conformational flexibilities possibly influence the binding modes of abiraterone.

In the X-ray crystal structure (pose A in Fig. 4A), abiraterone binds to Asn202 (F-helix) within CYP17A1 active site via H-bonding, whereas the roles of Arg239 and Asp298 were unclear (DeVore and Scott, 2012). It was observed during MD simulations that abiraterone adopts pose B when Arg239 (G-helix) and Asp298 (I-helix) are sufficiently close to form a salt bridge ( $\sim 3 \AA$ ) , but abiraterone tends to assume pose A as the distance between these residues increases. Tyr201 was found to be the most flexible side chain in the CYP17A1 binding site, with a root mean square fluctuation value of 2.8 $\AA$. The side chain dihedral angle of Tyr201, specifically $\chi 1$ (N-CA-CB-CG), repositions from a near gauche $\left(\sim 70^{\circ}\right)$ to a near trans $\left(\sim 170^{\circ}\right)$ conformation during simulations. This conformational rearrangement of Tyr201 favors the charge interaction between Arg239 and Asp298 and, hence, adoption of pose B. Thus, Tyr201, Arg239, and Asp298 play a crucial role in the reversible binding of abiraterone to CYP17A1. Collectively, MD simulations have provided insights into conformational adaptations within CYP17A1 that could justify the two predominant modes of abiraterone binding.

Apart from structural determinants, our findings underscore that equilibrium dissociation constants (thermodynamic selectivity) are not unequivocal indicators of the lifetime of drug-target complex (kinetic selectivity). This recognition has prompted the recent emergence of residence time $\left(1 / k_{\text {off }} \approx 1 / k_{4}\right)$ as the paramount indicator of PD efficacy (Copeland et al., 2006; Copeland, 2016; Tonge, 2018). Interpreting the prolonged residence time obtained for abiraterone in the context of previous dialysis and jump dilution studies demonstrates the validity of our estimates (Jarman et al., 1998; Garrido et al., 2014).

Nevertheless, the potential contribution of kinetic selectivity to in vivo efficacy remains complex because of dynamic changes in drug concentration, target turnover, and the relationship between target occupancy and effect (i.e., target vulnerability) (Daryaee and Tonge, 2019). Selection of a 500$\mathrm{mg}$ dose for comparison with the standard 1000-mg dose of AA was guided by results of a phase 1 single-dose study in castrate males in which target testosterone suppression was achieved and sustained for days 2-5 post-therapy (O'Donnell et al., 2004). Here, incorporating CYP17A1 turnover as well the plasma $\mathrm{PK}$ of abiraterone in relation to the lifetime of abiraterone-CYP17A1 complex, we first established that at both 1000- and 500-mg doses of AA, apparent protracted CYP17A1 engagement by abiraterone could be achieved in vivo (Fig. 6A). In subsequent PK-PD simulations, dynamic CYP17A1 engagement was correlated with in vitro androgen suppression. Interestingly, we further demonstrated comparable attenuations in intracellular DHEA-S levels associated with both 1000 and $500 \mathrm{mg}$ AA doses (Fig. 6C). Taken together, our kinetic selectivity approach facilitated rationalization of a clinically observed PK-PD uncoupling highlighted by Szmulewitz et al. (2018) and advocates a lower dosing requirement of abiraterone than that mandated by the MTD approach. Given that a 500-mg dose of AA administered fasted was shown to yield comparable PK with $250 \mathrm{mg}$ of AA administered with a low-fat meal (Fig. 7), dose reduction could represent a viable alternative to circumvent the high 


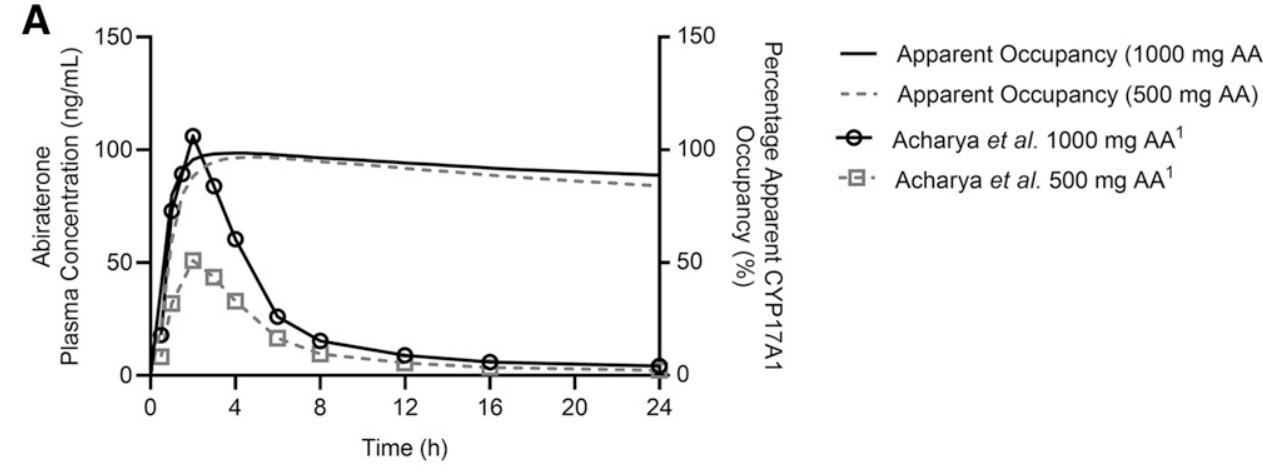

B

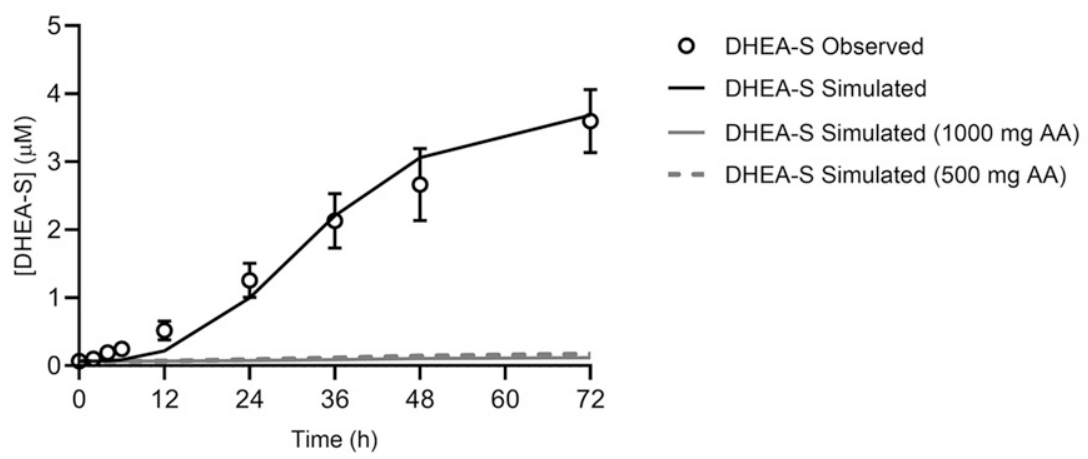

Fig. 6. Mechanistic pharmacokineticpharmacodynamic simulations integrating abiraterone PK and CYP17A1-abiraterone binding kinetics into eventual predictions of efficacy outcomes. (A) Simulated apparent CYP17A1 occupancy over time plots after a single in vivo dose of 1000 or $500 \mathrm{mg}$ of abiraterone acetate (AA). The clinical plasma concentration-time profiles of abiraterone are represented by open symbols, whereas the solid and dashed lines indicate the predicted percentage apparent CYP17A1 occupancy over time. Time-dependent changes in apparent CYP17A1 engagement were subsequently used to predict potential perturbations in the intracellular concentrations of (B) dehydroepiandrosterone-sulfate after multiple-dose administration of 1000 or $500 \mathrm{mg}$ AA over 72 hours. variability in oral bioavailability that often accompanies the food effect in drug PK.

With abiraterone slated for treating newly diagnosed highrisk $\mathrm{mHSPC}$, its average duration of treatment could span up to 24 months (Fizazi et al., 2017). Therefore, the pharmacoeconomic implications of any potential dose reduction could be substantial, as abiraterone acetate has an approximate retail cost of USD 10,000 per month (Szmulewitz et al., 2018). Moreover, the inhibition of the $17 \alpha$-hydroxylase reaction by abiraterone has been shown to produce dose-dependent mineralocorticoid-related toxicities that necessitates concomitant prednisone administration. Hence, potential dose reductions could also be instrumental in enhancing the safety of abiraterone therapy.

It is important to note that apparent occupancy-time profiles (Fig. 6A) were generated on the assumption of equivalent total tissue and plasma concentrations. This approach deviates from that advocated by the free drug hypothesis, in which drug molecules bind to plasma and tissue proteins, resulting in a reduction in free, pharmacologically active concentrations necessary for target engagement. Based on the free drug theory, with assumptions of steady-state PK and the dominance of passive diffusion processes, the unbound partition coefficient $\left(\mathrm{K}_{\mathrm{p}, \mathrm{uu}}\right)$ for a noneliminating tissue will equal unity $\left(\mathrm{C}_{\mathrm{u}, t} / \mathrm{C}_{\mathrm{u}, \mathrm{p}}=\sim 1\right)$ (Zhang et al., 2019). Expectedly, when unbound plasma concentrations were used as a surrogate for unbound tissue concentrations to effect CYP17A1 engagement, the extent of CYP17A1 occupancy over time was significantly diminished (Supplemental Fig. 8A). Nevertheless, in the case of abiraterone, we believe that asymmetry of free drug concentrations between target tissues and plasma at steady state is a distinct possibility. Firstly, corroborating Mostaghel et al. (2017), our preliminary analyses demonstrated potential organic anion transporting polypeptide 1B3-mediated abiraterone uptake (Supplemental Fig. 8B).
Coupled with evidence supporting increased expression of organic anion transporting polypeptide 1B3 in metastatic lesions from prostate cancer, intratumoral levels of abiraterone could be reasonably higher than that predicted by $K_{p \text {,uu }}$ (Schulte and Ho, 2019). Additionally, another key assumption underpinning the free drug hypothesis is that interactions between the drug and the receptor are reversible. Consequently, concentration gradient can be the sole factor invoked to describe free drug permeation. However, with the tightbinding of abiraterone to CYP17A1, it is possible that with adequate abiraterone concentrations exceeding the target covalent binding threshold, a separate concentration gradient can be re-established with the excess free abiraterone (Zhang et al., 2019). This postulation is also supported by distribution studies performed in rats, in which the highest ${ }^{14} \mathrm{C}$-abiraterone concentrations detected within the adrenal gland were 1535 times the corresponding blood concentration (European Medicines Agency, 2011). Taken together, there remains considerable uncertainties in occupancy determinations because of the inability to accurately estimate the molar ratio of intracellular free abiraterone versus CYP17A1. As such, the threshold and strength of correlation between target occupancy and effect as described by a target vulnerability function remains poorly defined. Consequently, direct quantification of in vivo target engagement via imaging techniques such as positron emission tomography becomes imperative to inform and correct estimates of apparent CYP17A1 occupancy.

In the case of $\mathrm{D} 4 \mathrm{~A}$, residence time effects were also expected, given the prolonged CYP17A1 engagement observed in in vitro kinetic analyses. Nevertheless, prolongation of phenotypic response after D4A washout was not observed (data not shown). This apparent incongruity could be attributed to the susceptibility of the $\Delta 4,3$-keto structure of $\mathrm{D} 4 \mathrm{~A}$ to irreversible $5 \alpha$-reduction and $5 \beta$-reduction within the tumor microenvironment ( $\mathrm{Li}$ et al., 2016). In fact, direct incubation of 
A

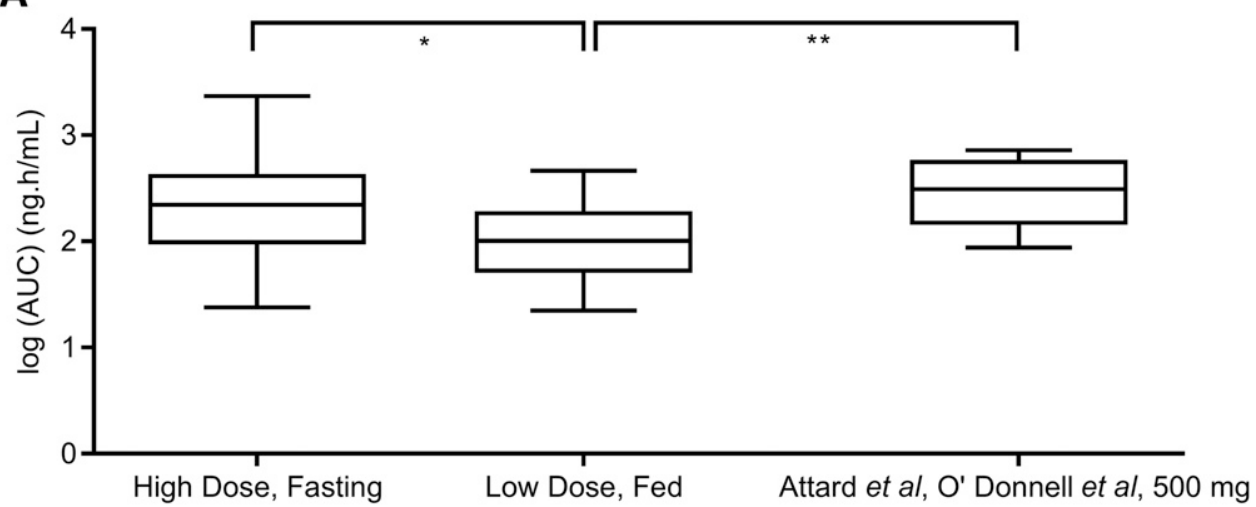

B

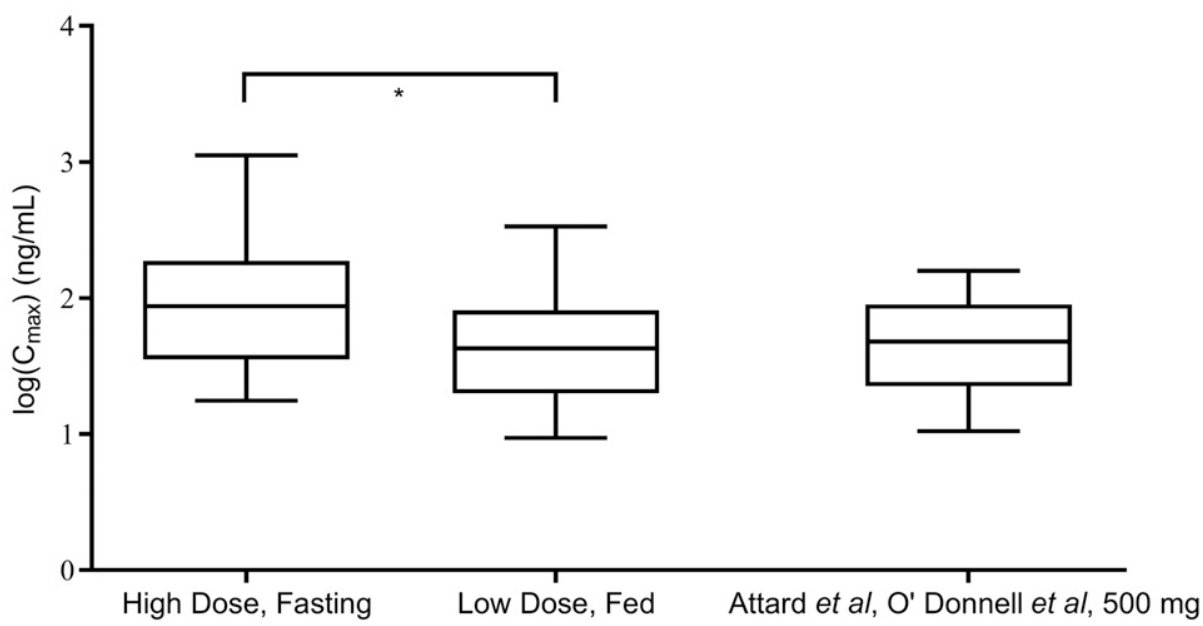

Fig. 7. Investigating potential correlations between abiraterone drug levels and the extent of DHEA-S suppression. Results from the phase II study by Szmulewitz et al. (2018) demonstrated how (A) the area under the plasma concentration-time curve of abiraterone from 0 to 4 hours $\left(\mathrm{AUC}_{0-4}\right.$ hours $)$, and (B) peak plasma concentrations $\left(C_{\text {max }}\right)$ remained significantly higher in the standard-dose arm (1000 mg AA, fasting, $n=20$ ) compared with the low-dose arm (250 mg AA, fed, $n=20$ ) (two-sided $P$ value for $\mathrm{AUC}_{0-4}$ hours $=0.0417$, and twosided $P$ value for $\left.C_{\max }=0.0474\right)$. Combined analyses of phase I trials (Attard et al., 2008; O'Donnell et al., 2004, $n=10$ ) in (A and $\mathrm{B}$ ) revealed that administration of $500 \mathrm{mg}$ of AA in a fasted state yielded AUC values that were greater than that measured in the low-dose arm (two-sided $P$ value $=0.0038$ ), whereas $C_{\max }$ measurements were not significantly different. AUC and $C_{\text {max }}$ data were log-transformed and groups comparisons were performed using repeated-measures one-way ANOVA and Dunnett's multiple comparisons tests.
D4A in LAPC4 prostate cancer cell line for 48 hours demonstrated substantial downstream conversion of D4A to the initial $5 \alpha$-reduced metabolite (3-keto- $5 \alpha$-abiraterone), which is similarly present at higher concentrations than D4A in patients with mCRPC taking abiraterone ( $\mathrm{Li}$ et al., 2016). Hence, it is possible that the elimination of $\mathrm{D} 4 \mathrm{~A}$ is not ratelimiting, diminishing the utility of a prolonged residence time in extending its PD durability (Dahl and Akerud, 2013).

In conclusion, this study provides compelling evidence that abiraterone is a slow-, tight-binding inhibitor of steroidogenic CYP17A1. Our in vitro enzyme kinetic analyses provided quantitative description of pertinent kinetic parameters characterizing the two-step binding that are entirely consistent with the results of MD simulations. Cellular washout experiments provided further corroborative evidence for the protracted residence time of abiraterone on CYP17A1. In addition, MD simulations offered insights into the fast, reversible step preceding tight-binding that was not apparent from either X-ray crystallography or molecular docking simulations. The long dissociation $t_{1 / 2}$ of both abiraterone and $\mathrm{D} 4 \mathrm{~A}$ reflects protracted residence time on CYP17A1, which in turn creates the awareness that the incorporation of residence time along with PK parameters is imperative in the mechanistic PK-PD modeling of abiraterone to optimize mCRPC pharmacotherapy.

\section{Acknowledgments}

This research was undertaken with the assistance of resources from the National Computational Infrastructure, which is supported by the Australian government. The authors also acknowledge Flinders University's High Performance Computing resources for this work.

\section{Authorship Contributions}

Participated in research design: Cheong, Nair, Neo, Tu, Lin, Fan, Miners, Chan.

Conducted experiments: Cheong, Nair, Neo, Tu, Lin, Fan, Miners, Chan.

Contributed new reagents or analytic tools: Tu, Chai.

Performed data analysis: Cheong, Nair, Neo, Tu, Lin, Fan, Szmulewitz, Peer, Figg, Miners, Chan.

Wrote or contributed to the writing of the manuscript: Cheong, Nair, Neo, Tu, Lin, Chiong, Esuvaranathan, Fan, Chai, Miners, Chan.

\section{References}

Abraham MJ, Murtola T, Schulz R, Páll S, Smith JC, Hess B, and Lindahl E (2015) GROMACS: high performance molecular simulations through multi-level parallelism from laptops to supercomputers. SoftwareX 1-2:19-25.

Acharya M, Bernard A, Gonzalez M, Jiao J, De Vries R, and Tran N (2012) Openlabel, phase I, pharmacokinetic studies of abiraterone acetate in healthy men. Cancer Chemother Pharmacol 69:1583-1590.

Ahmed KEM, Frøysa HG, Karlsen OD, Sagen JV, Mellgren G, Verhaegen S, Ropstad E, Goksøyr A, and Kellmann R (2018) LC-MS/MS based profiling and dynamic modelling of the steroidogenesis pathway in adrenocarcinoma H295R cells. Toxicology in Vitro 52:332-341.

Attard G, Reid AHM, Yap TA, Raynaud F, Dowsett M, Settatree S, Barrett M, Parker C, Martins V, Folkerd E, et al. (2008) Phase I clinical trial of a selective inhibitor of CYP17, abiraterone acetate, confirms that castration-resistant prostate cancer commonly remains hormone driven. J Clin Oncol 26:4563-4571.

Bray F, Ferlay J, Soerjomataram I, Siegel RL, Torre LA, and Jemal A (2018) Global cancer statistics 2018: GLOBOCAN estimates of incidence and mortality worldwide for 36 cancers in 185 countries. CA Cancer J Clin 68:394-424.

Chen R and Chung S-H (2012) Binding modes and functional surface of antimammalian scorpion $\alpha$-toxins to sodium channels. Biochemistry 51:7775-7782. 
Copeland RA (2013) Slow binding inhibitors, Evaluation of Enzyme Inhibitors in Drug Discovery pp 203-244, John Wiley \& Sons, Inc, Hoboken NJ.

Copeland RA (2016) The drug-target residence time model: a 10-year retrospective. Nat Rev Drug Discov 15:87-95.

Copeland RA (2000) Time-dependent inhibition, Enzymes: A Practical Introduction to Structure Mechanism, and Data Analysis pp 318-349, Wiley, New York.

Copeland RA, Pompliano DL, and Meek TD (2006) Drug-target residence time and its implications for lead optimization. Nat Rev Drug Discov 5:730-739.

Dahl G and Akerud T (2013) Pharmacokinetics and the drug-target residence time concept. Drug Discov Today 18:697-707.

Darden TA, York DM, and Pedersen LG (1993) Particle Mesh Ewald: An Nlog (N) Method for Ewald Sums in Large Systems. The Journal of Chemical Physics 98: 10089-10092.

Daryaee F and Tonge PJ (2019) Pharmacokinetic-pharmacodynamic models that incorporate drug-target binding kinetics. Curr Opin Chem Biol 50:120-127.

DeVore NM and Scott EE (2012) Structures of cytochrome P450 17A1 with prostate cancer drugs abiraterone and TOK-001. Nature 482:116-119.

Ekroos M and Sjögren T (2006) Structural basis for ligand promiscuity in cytochrome P450 3A4. Proc Natl Acad Sci USA 103:13682-13687.

Essmann U, Perera L, Berkowitz ML, Darden T, Lee H, and Pedersen LG (1995) A smooth particle mesh Ewald method. J Chem Phys 103:8577-8593.

European Medicines Agency (2011) Assessment report for Zytiga (abiraterone).

European Medicines Agency (2017) EMA/816845/2017 - Assessment Report, Zytiga https://www.ema.europa.eu/en/documents/variation-report/zytiga-h-c-2321-ii-0047epar-assessment-report-variation_en.pdf.

Fizazi K, Tran N, Fein L, Matsubara N, Rodriguez-Antolin A, Alekseev BY, Özgüroğlu M, Ye D, Feyerabend S, Protheroe A, et al.; LATITUDE Investigators (2017) Abiraterone plus prednisone in metastatic, castration-sensitive prostate cancer. N Engl J Med 377:352-360.

Garrido M, Peng HM, Yoshimoto FK, Upadhyay SK, Bratoeff E, and Auchus RJ (2014) A-ring modified steroidal azoles retaining similar potent and slowly reversible CYP17A1 inhibition as abiraterone. J Steroid Biochem Mol Biol 143:1-10.

Ghosh D, Egbuta C, and Lo J (2018) Testosterone complex and non-steroidal ligands of human aromatase. J Steroid Biochem Mol Biol 181:11-19.

Gordon D, Chen R, and Chung S-H (2013) Computational methods of studying the binding of toxins from venomous animals to biological ion channels: theory and applications. Physiol Rev 93:767-802.

Harris WP, Mostaghel EA, Nelson PS, and Montgomery B (2009) Androgen deprivation therapy: progress in understanding mechanisms of resistance and optimizing androgen depletion. Nat Clin Pract Urol 6:76-85.

Hoover WG (1985) Canonical dynamics: equilibrium phase-space distributions. Phys Rev A Gen Phys 31:1695-1697.

Hub JS, de Groot BL, and van der Spoel D (2010) g_wham-a free weighted histogram analysis Implementation including robust error and autocorrelation estimates. J Chem Theory Comput 6:3713-3720.

Janssen (2019) ZYTIGA® (abiraterone acetate) [Highlights of Prescribing Information].

Jarman M, Barrie SE, and Llera JM (1998) The 16,17-double bond is needed for irreversible inhibition of human cytochrome p45017alpha by abiraterone (17-(3pyridyl)androsta-5, 16-dien-3beta-ol) and related steroidal inhibitors. J Med Chem 41:5375-5381.

Ji Y, Jin JY, Hyman DM, Kim G, and Suri A (2018) Challenges and opportunities in dose finding in oncology and immuno-oncology. Clin Transl Sci 11:345-351.

Kumar S, Rosenberg JM, Bouzida D, Swendsen RH, and Kollman PA (1992) THE weighted histogram analysis method for free-energy calculations on biomolecules. I. The method. J Comput Chem 13:1011-1021.

Li Z, Alyamani M, Li J, Rogacki K, Abazeed M, Upadhyay SK, Balk SP, Taplin M-E, Auchus RJ, and Sharifi N (2016) Redirecting abiraterone metabolism to fine-tune prostate cancer anti-androgen therapy. Nature 533:547-551.

Li Z, Bishop AC, Alyamani M, Garcia JA, Dreicer R, Bunch D, Liu J, Upadhyay SK, Auchus RJ, and Sharifi N (2015) Conversion of abiraterone to D4A drives antitumour activity in prostate cancer. Nature 523:347-351.

Malde AK, Zuo L, Breeze M, Stroet M, Poger D, Nair PC, Oostenbrink C, and Mark AE (2011) An automated force field Topology builder (ATB) and repository: version 1.0. J Chem Theory Comput 7:4026-4037.

Minasian L, Rosen O, Auclair D, Rahman A, Pazdur R, and Schilsky RL (2014) Optimizing dosing of oncology drugs. Clin Pharmacol Ther 96:572-579.

Ming DS, Pham S, Deb S, Chin MY, Kharmate G, Adomat H, Beheshti EH, Locke J, and Guns ET (2014) Pomegranate extracts impact the androgen biosynthesis pathways in prostate cancer models in vitro and in vivo. $J$ Steroid Biochem Mol Biol 143:19-28.

Morrison JF and Walsh CT (1988) The behavior and significance of slow-binding enzyme inhibitors, in Advances in Enzymology and Related Areas of Molecular Biology (Meister A 201-301, John Wiley \& Sons, Inc., New York.
Mostaghel EA, Cho E, Zhang A, Alyamani M, Kaipainen A, Green S, Marck BT, Sharifi N, Wright JL, Gulati R, et al. (2017) Association of tissue abiraterone levels and SLCO genotype with intraprostatic steroids and pathologic response in men with high-risk localized prostate cancer. Clin Cancer Res 23:4592-4601.

Mostaghel EA, Page ST, Lin DW, Fazli L, Coleman IM, True LD, Knudsen B, Hess DL, Nelson CC, Matsumoto AM, et al. (2007) Intraprostatic androgens and androgenregulated gene expression persist after testosterone suppression: therapeutic implications for castration-resistant prostate cancer. Cancer Res 67:5033-5041.

Nair PC, McKinnon RA, and Miners JO (2016) Cytochrome P450 structure-function: insights from molecular dynamics simulations. Drug Metab Rev 48:434-452.

Nosé S (1984) A unified formulation of the constant temperature molecular dynamics methods. J Chem Phys 81:511-519.

O'Donnell A, Judson I, Dowsett M, Raynaud F, Dearnaley D, Mason M, Harland S, Robbins A, Halbert G, Nutley B, et al. (2004) Hormonal impact of the $17 \alpha$-hydroxylase/C(17,20)-lyase inhibitor abiraterone acetate (CB7630) in patients with prostate cancer. $\mathrm{Br} J$ Cancer 90:2317-2325.

Parrinello M and Rahman A (1981) Polymorphic transitions in single crystals: a new molecular dynamics method. J Appl Phys 52:7182-7190.

Petrunak EM, DeVore NM, Porubsky PR, and Scott EE (2014) Structures of human steroidogenic cytochrome P450 17A1 with substrates. $J$ Biol Chem 289: 32952-32964.

Petrunak EM, Rogers SA, Aubé J, and Scott EE (2017) Structural and functional evaluation of clinically relevant inhibitors of steroidogenic cytochrome P450 17A1. Drug Metab Dispos 45:635-645.

Pienta KJ and Bradley D (2006) Mechanisms underlying the development of androgen-independent prostate cancer. Clin Cancer Res 12:1665-1671.

Porubek D (2013) CYP17A1: a biochemistry, chemistry, and clinical review. Curr Top Med Chem 13:1364-1384.

R Core Team (2013) R: A Language and Environment for Statistical Computing, R Foundation for Statistical Computing, Vienna, Austria.

Sachs JR, Mayawala K, Gadamsetty S, Kang SP, and de Alwis DP (2016) Optimal dosing for targeted therapies in oncology: drug development cases leading by example. Clin Cancer Res 22:1318-1324.

Salmaso V and Moro S (2018) Bridging molecular docking to molecular dynamics in exploring ligand-protein recognition process: an overview. Front Pharmacol 9:923.

Scher HI, Morris MJ, Stadler WM, Higano C, Basch E, Fizazi K, Antonarakis ES, Beer TM, Carducci MA, Chi KN, et al.; Prostate Cancer Clinical Trials Working Group 3 (2016) Trial design and objectives for castration-resistant prostate cancer: updated recommendations from the prostate cancer clinical trials working group 3 . $J$ Clin Oncol 34:1402-1418.

Schmid N, Eichenberger AP, Choutko A, Riniker S, Winger M, Mark AE, and van Gunsteren WF (2011) Definition and testing of the GROMOS force-field versions 54A7 and 54B7. Eur Biophys $J$ 40:843-856.

Schmidtke P, Bidon-Chanal A, Luque FJ, and Barril X (2011) MDpocket: open-source cavity detection and characterization on molecular dynamics trajectories. Bioinformatics 27:3276-3285.

Schulte RR and Ho RH (2019) Organic anion transporting polypeptides: emerging roles in cancer pharmacology. Mol Pharmacol 95:490-506.

Sevrioukova IF and Poulos TL (2017) Structural basis for regiospecific midazolam oxidation by human cytochrome P450 3A4. Proc Natl Acad Sci USA 114:486-491.

Szegezdi J and Csizmadia F (2007) Calculating the pKa values of small and large molecules, in American Chemical Society Spring meeting.

Szmulewitz RZ, Peer CJ, Ibraheem A, Martinez E, Kozloff MF, Carthon B, Harvey RD, Fishkin P, Yong WP, Chiong E, et al. (2018) Prospective international randomized phase II study of low-dose abiraterone with food versus standard dose abiraterone in castration-resistant prostate cancer. J Clin Oncol 36:1389-1395.

Tonge PJ (2018) Drug-target kinetics in drug discovery. ACS Chem Neurosci 9:29-39.

Torrie GM and Valleau JP (1977) Nonphysical sampling distributions in Monte Carlo free-energy estimation: umbrella sampling. J Comput Phys 23:187-199.

Vasaitis TS, Bruno RD, and Njar VCO (2011) CYP17 inhibitors for prostate cancer therapy. J Steroid Biochem Mol Biol 125:23-31.

Vauquelin G (2017) Distinct in vivo target occupancy by bivalent- and induced-fit-like binding drugs. Br J Pharmacol 174:4233-4246.

Yin L and $\mathrm{Hu}$ Q (2014) CYP17 inhibitors-abiraterone, C17,20-lyase inhibitors and multi-targeting agents. Nat Rev Urol 11:32-42.

Zhang D, Hop CECA, Patilea-Vrana G, Gampa G, Seneviratne HK, Unadkat JD, Kenny JR, Nagapudi K, Di L, Zhou L, et al. (2019) Drug concentration asymmetry in tissues and plasma for small molecule-related therapeutic modalities. Drug Metab Dispos 47:1122-1135.

Address correspondence to: Eric Chun Yong Chan, Department of Pharmacy, Faculty of Science, National University of Singapore, 18 Science Drive 4, Singapore 117543, Singapore. E-mail: phaccye@nus.edu.sg 